\title{
SIS/aligned fibre scaffold designed to meet layered oesophageal tissue complexity and properties
}

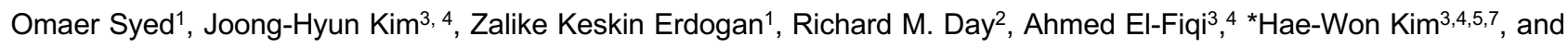
*Jonathan C. Knowles $1,3,6,7$

${ }^{1}$ Division of Biomaterials and Tissue Engineering, University College London Eastman Dental Institute, 256 Grays Inn Road, London WC1X 8LD, UK

${ }^{2}$ Applied Biomedical Engineering Group, Division of Medicine, University College London, 5 University St, London WC1E 6JF

${ }^{3}$ Department of Nanobiomedical Science \& BK21 PLUS NBM Global Research Center for Regenerative Medicine, Dankook University, Cheonan 31116, Republic of Korea

${ }^{4}$ Institute of Tissue Regeneration Engineering (ITREN), Dankook University, Cheonan 31116, Republic of Korea

${ }^{5}$ Department of Biomaterials Science, College of Dentistry, Dankook University, Cheonan 31116, Republic of Korea

${ }^{6}$ The Discoveries Centre for Regenerative and Precision Medicine, UCL Campus, London, UK

${ }^{7}$ UCL Eastman-Korea Dental Medicine Innovation Centre, Dankook University, Cheonan 31116, Republic of Korea

*Co-corresponding authors: j.knowles@ucl.ac.uk and kimhw@dku.edu

\section{Abstract}

With donor organs not readily available, the need for a tissue engineered oesophagus remains high, particularly for congenital childhood conditions such as atresia. Previous attempts have not been successful and challenges remain. Small intestine submucosa (SIS) is an acellular matrix material with good biological properties though, as is common with these types of materials, demonstrably poor mechanical properties. In this work, electrospinning was used to mechanically reinforce tubular SIS with poly lactic-co-glycolic acid PLGA nanofibers. It was hypothesised that if attachment could be achieved between the two materials this would (i) improve the SIS mechanical properties, (ii) facilitate smooth muscle cell alignment to support directional growth of muscle cells and (iii) allow for the delivery of bioactive molecules (VEGF in this instance).

Through a relatively simple multistage process, adhesion between the layers was achieved without chemically altering the SIS. It was also found that altering mandrel rotation speed affected the alignment of the PLGA nanofibers. SIS-PLGA scaffolds performed mechanically better than SIS alone; yield stress improvement was $200 \%$ and $400 \%$ along the longitudinal and circumferential direction, respectively. Smooth muscle cells cultured on the aligned fibres showed resultant unidirectional alignment. In vivo the SIS-PLGA scaffolds demonstrated limited foreign body reaction judged by the type and proportion of immune cells present and lack of fibrous encapsulation. The scaffolds remained intact at 4 weeks, in vivo and good cellular infiltration was observed. The incorporation of VEGF within SIS-PLGA scaffolds increased the blood vessel density of the surrounding tissues, highlighting the possible stimulation of endothelialisation by angiogenic factor delivery. Overall, the designed SIS-PLGA-VEGF hybrid scaffolds might be used as a potential matrix platform for oesophageal tissue engineering. In addition to this, achieving improved attachment between layers of acellular matrix materials and electrospun fibres layers offers the potential utility in other applications.

Keywords: Electrospinning, PLGA, Oesophageal tissue, acellular organ matrix

\section{Introduction}

Medical conditions which affect the oesophageal region often require surgical intervention as a treatment. This is the case for both acquired conditions, such as cancer or physical injury, and congenital ones, such as atresia [1]. Complicated surgical procedures are often required, such as gastric transposition or intestinal interposition [2], with associated post- 
operative complications including stricture formation, anastomotic leakages, dysphagia, dysmotility and gastrooesophageal reflux $[3,4]$. Therefore, tissue-engineering may represent a promising alternative strategy.

A number of different tissue engineering approaches have been attempted and have been reviewed elsewhere [5, 6]. These attempts have had limited success with particular difficulty in regrowing muscular tissue. One material used in these attempts was SIS, which has a number of desirable properties specifically related to limited immune reaction and rapid remodelling [7-11]. In one early approach to oesophageal tissue engineering SIS was sutured into a tube for segmental replacement in a canine model, however the in vivo results were not satisfactory with stenosis and detachment being the major causes of failure, and the tissue was largely devoid of muscle cells as with other approaches [12]. When SIS in sheet form was used in oesophageal repair in nonsegmental "patches" in animal models the SIS did integrate well, but issues with mechanical properties of the materials were thought to contribute to the problems observed [13, 14]. It was suggested that if a stent-like structure was present during the initial three months, (the period when the majority of failures occurred), some of the observed failures could be prevented [12].

SIS has already been shown to be readily decellularised and processed into a tubular scaffold [15]. It was hypothesized that a fibrous electrospun layer could be used to reinforce the SIS, and function as a stent while the material undergoes the remodelling phase as it is during this phase that mechanical properties have been observed to be insufficient in the unreinforced SIS [16]. A number of groups have successfully shown that electrospun fibres can be used to produce constructs [17] including tubular ones [18]. It has also been demonstrated that electrospun fibres can adhere to decellularised ECM materials under certain conditions [19].

PLGA, was chosen as it is one of the most commonly used synthetic materials for preparing fibrous scaffolds in tissue engineering and has a good track record in clinical use. Furthermore the degradation time and mechanical properties can be adjusted [20]. PLGA electrospun fibrous scaffolds have drawn singificant attention in the area of tissue engineering and drug delivery system due to its well-known good processability and good mechanical performance.

In this study, we investigated whether an electrospun layer can successfully reinforce a tubular SIS scaffold to produce a hybrid scaffold for oesophageal tissue engineering. The current design is schematically illustrated in Figure 1 . The electrospun fibre/SIS hybrid scaffold is envisaged to function without cells for short-length replacement and repair; however for longer-segments cells can be populated onto the scaffold. Given the lack of muscle development in previous attempts it is proposed that smooth muscle cells can be cultured onto the scaffold in vitro. The alignment of electrospun fibres is also considered an important matrix parameter [20, 21]. In fact, for vascular tissue engineering, the fibre alignment was effective for smooth muscle cell alignment with the aim of ultimately restoring contractility [20, 22]. A similar approach was adopted in this study for oesophageal tissue engineering. The electrospun fibre scaffolds also present an opportunity to facilitate therapeutic molecule delivery. Vascular endothelial growth factor (VEGF) was chosen due to it being a potent angiogenic factor for endothelialisation [23], a critical event to fully enable vascularized oesophageal tissue engineering. 
Figure 1 Schematic illustrating the various stages of the development of hybrid PLGA-SIS scaffolds and potential applications for oesophageal tissue engineering 


\section{Materials and Methods}

\subsection{SIS Preparation}

Porcine small intestine was obtained from a local abattoir from animals 6 months in age. The jejunum/ileum samples were cut to $20 \mathrm{~cm}$ lengths, trimmed of excess mesentery tissue and then thoroughly rinsed with deionised water (DI) to remove debris. The small intestine submucosa (SIS) was isolated using minor modifications of the well-established method $[24,25]$. The lengths of small intestine were inverted and then gently abraded using moist surgical gauze to remove the inner mucosal layer. After thoroughly washing with deionised water, the tissue was then re-inverted and the serosal layer manually removed following a small incision with a scalpel. Finally the outer surface was carefully abraded using damp gauze to remove any additional remnants of the outer layers. The SIS was then carefully washed twice in deionised water for 5 minutes, which involved stirring and frequent replacement of fresh deionised water. The SIS was then stored in PBS (phosphate buffered saline: $0.01 \mathrm{M}$ phosphate buffer $0.0027 \mathrm{M}$ potassium chloride and $0.137 \mathrm{M}$ sodium chloride, $\mathrm{pH} 7.4$, at $25^{\circ} \mathrm{C}$ ) at $4^{\circ} \mathrm{C}$.

\subsection{Decellularisation \& Processing}

The lengths of SIS were connected to tubing in individual sealable containers filled with $350 \mathrm{ml}$ of $4 \%$ sodium deoxycholate (Sigma-Aldrich Company Ltd., Dorset, UK), and then perfusion was carried out at $500 \mathrm{ml} / \mathrm{h}$ for $12 \mathrm{~h}$ at RT. The samples were removed and thoroughly rinsed with DI, and then a perfusion wash was carried out for 15 min, with uktrapure water. A final wash cycle was carried out for $6 \mathrm{~h}$ using non-cycled deionised water from separate reservoir. The treated SIS was stretched over a $2.54 \mathrm{~cm}$ diameter (1 inch) porous polyethylene tubing (60 $\mu \mathrm{m}$ pores) (Porex Corporation, Fairburn, USA), (an earlier version of SIS shown in Figure 4a used tubing with $1.9 \mathrm{~cm}$ diameter). The SIS was then vacuum-dried in sealed sterile specimen bags under low vacuum conditions (approximately $10 \mathrm{mBar}$ ) for $12 \mathrm{~h}$ to compress and dry the samples. The dried SIS samples were then lyophilised by $1 \mathrm{~min}$ of immersion in liquid nitrogen followed by $12 \mathrm{~h}$ in a vacuum freeze-drier (Heto Dry Winner). These stages and the final appearance of the processed SIS are shown in supplementary figure 1. Levels of decellularisation were confirmed by DNA quantification and nuclei counting (Supplementary figure 2 and 3 ).

\subsection{Electrospinning}

The polymer used was poly(lactic-co-glycolic acid) (PLGA) (Purac ${ }^{\circledR}$ PLG 8523, 85/15 L-lactide/glycolide copolymer) (Corbion, Amsterdam, The Netherlands). The polymer granules were dissolved weight by volume to make a $15 \%$ solution using a solvent mixture of 8:2 dichloromethane (DCM) to dimethylformamide (DMF). The polymer solution was stored at $4{ }^{\circ} \mathrm{C}$. A conventional electrospinning rig was used with an 18 gauge flat ended needle above a rotating mandrel. The tubular SIS was cut to lengths of $3 \mathrm{~cm}$ and fixed to the mandrel. The electrospinning was carried out in 2 stages; the first being a thin coating layer with polymer solution pumped at a flow rate of $7 \mathrm{ml} \mathrm{h}^{-1}$ for 2 min at a voltage of $20 \mathrm{kV}$ and with the mandrel rotating at $10 \mathrm{rpm}$, this was followed the main layer with polymer solution pumped at a flow rate of $1 \mathrm{ml} \mathrm{h}^{-1}$ and at a voltage of $14 \mathrm{kV}$ for $45 \mathrm{~min}$. The mandrel was advanced along the longitudual axis at an increment of $1 \mathrm{~cm}$ every 15 minutes. The final scaffold samples were vacuum dried to remove any excess solvent. Samples were produced in 3 
groups based on the speed of mandrel rotation and representing different fibre orientations, named: 10 rpm (unaligned), $3000 \mathrm{rpm}$ (partially aligned) and $5000 \mathrm{rpm}$ (highly aligned). The setup used is shown in supplementary figures 4 and 5 .

\subsection{Stability testing of SIS/Fibre scaffold layers}

Samples of the material were prepared identically as those for mechanical testing i.e. cutting rectangular shapes of dimensions 8 - $14 \mathrm{~mm}$ in length and $5-8 \mathrm{~mm}$ in width. These were submerged in deionised water and placed on an orbital shaker set at $60 \mathrm{rpm}$ for $2 \mathrm{~h}$ at room temperature. The samples were removed and place on absorbent paper towels and dried using reasonable manual force (but with care taken to apply no torsional forces) and allowed to dry for $2 \mathrm{~h}$. The samples were then re-submerged in deionised water. Any separation of the layers was noted at any stage.

\subsection{Mechanical Testing of SIS/fibre aligned scaffold}

Uniaxial tensile testing was carried out using a dynamic mechanical analyser (DMA 7e, Perkin-Elmer Instruments, USA). Test sections were cut into rectangular shapes of dimensions 8-14 $\mathrm{mm}$ in length and 5-8 mm in width, and in both the longitudinal and circumferential directions (relative to the axis of the mandrel). All samples were rehydrated with uktrapure water for $30 \mathrm{~min}$, prior to testing. The ends of the samples were enclosed in wire gauze to provide grip and stability and the samples were then clamped into the machine. The dimensions of the samples were entered into the software (Pyris) and the distance between the two clamps was taken as the length. The tensile test was run with an increasing force value of $500 \mathrm{mN} \mathrm{min} \mathrm{m}^{-1}$. From the stress-strain graph produced the elastic modulus was calculated as the gradient of the elastic region of the curve ( $E=$ stress/strain) (Also see supplementary figure 6). Samples which slipped or failed near to the clamps were ignored.

\subsection{Scanning Electron Microscopy}

Samples were cut from the scaffold groups into squares $1 \times 1 \mathrm{~cm}$ and mounted on aluminium stubs using fast drying epoxy adhesive. The stubs were placed in a desiccator cabinet for at least $12 \mathrm{~h}$ to remove any moisture and then sputter coated with gold/palladium (Polaron E5000, Quorum Technology, UK). Imaging was carried out using a scanning electron microscope (Philips XL 30 SFEG, Netherland). Digital images produced were analysed using software (ImageJ).

Electrospun fibre analysis was performed using the Directionality v2.01 plugin for ImageJ which measured alignment angles on the SEM images. A minimum of 10 images were analysed for each scaffold group and care was taken to ensure the samples were correctly aligned prior to imaging. In images with lower magnification the images were cut into multiple images to improve the analysis using ImageJ. For comparative analysis the alignment of the fibres were grouped in 3 equal groups spanning 0 - $90^{\circ}$ from the mandrel axis of rotation.

\subsection{Porosity of SIS/fibre scaffolds}

The scaffolds were cut into samples of $1 \mathrm{~g}$ weight, rolled into a consistent shape, and were placed into the glass testing vessel of a mercury intrusion porosimeter (Micromeritics Autopore, Micromeritics Instrument Corporation, Norcross, USA). The porosity measurements were carried out using an automated testing cycle ( $n=$ between 4 and 6$)$. Statistical 
testing was carried out using a 2-tailed student's t-test to a 95\% confidence interval using SPSS version 20 (IBM, Armonk, New York, US).

\subsection{Human oesophageal smooth muscle cell culture}

Primary human oesophageal smooth muscle cells (HESMC, ScienCell Research Laboratories, Carlsbad, CA, USA) were cultured in Dulbecco's modified Eagle medium (Gibco, Life Technologies, Paisley, UK) supplemented with 10\% foetal bovine serum (Gibco) and $1 \%$ penicillin-streptomycin (Gibco) at $37^{\circ} \mathrm{C}$ with $5 \% \mathrm{CO}_{2}$ and $95 \%$ relative humidity. Cells from passages 2 - 6 were used for experiments. A CellTiter 96 AQueous Non-Radioactive Cell Proliferation Assay (Promega, Southampton, UK) was used to assess the metabolic activity of HESMC's as an indication of biocompatibility, in accordance with ISO 10993 parts 5 and 12 and the manufacturer's protocol. Briefly, HEMSC were seeded at a density of 100,000 cells $/ \mathrm{cm}^{2}$ on the scaffolds with the PLGA fibres spun at $10 \mathrm{rpm}, 1000 \mathrm{rpm}, 3000 \mathrm{rpm}$, 5000 rpm, a commercial scaffold Biodesign ${ }^{\circledR}$ (Cook Medical, UK) and as a control tissue culture plates (TCP 48-wells) were used. Cell culture inserts (CellCrown, Scaffdex Ltd., Tampere, Finland) were used to pull the samples taut and suspend them within the 24-well plates with the cells seeded on the PLGA nanofibre surface. For sterilisation the scaffolds were immersed in a $70 \%$ ethanol solution and then allowed to dry completely in a tissue culture cabinet. The scaffolds were all hydrated with $200 \mu$ l of growth medium for 30 min prior to cell seeding.

\subsection{Viability of HESMC cultured on SIS/aligned fibre scaffold}

After 24 and 96 hours incubation, medium was aspirated from the cells and replaced with one part MTS solution in six parts medium, which consists of (3-(4, 5-dimethylthiazol-2-yl)-5-(3-carboxymethoxyphenyl)-2-(4-sulfophenyl)-2Htetrazolium) (MTS dye) and electron coupling agent phenazine methosulfate. After 1 - $1.5 \mathrm{~h}$ incubation, absorbance was measured at $490 \mathrm{~nm}$, with a reference wavelength of $630 \mathrm{~nm}$ at each time point using a spectrophotometer (M200, Tecan, Männedorf / Switzerland). The plates were measured along with a standard plate of known cell concentrations prepared for each time-point. Between time-points, MTS-containing medium was aspirated, the cells rinsed with PBS, and replaced with fresh growth medium. The metabolic activity of the cells was normalised to the TCP control at the end of experiment.

\subsection{Live/dead Cell Assay HESMC cultured on SIS/aligned fibre scaffold}

The scaffolds (10 rpm, 1000 rpm, 3000 rpm and 5000 rpm) and the commercial SIS (Biodesign ${ }^{\circledR}$ Cook Medical,UK) were prepared as described previously and placed in identical arrangements using 24-well plates and CellCrown inserts. The control wells used in this instance were 24-well plates, with sterile glass coverslip inserts. HEMSC were seeded at a density of 100,000 cells $/ \mathrm{cm}^{2}$. The cells were cultured for 1 day, 7 days and 14 days. At the relevant time points the medium was removed from the wells and a mixture of Calcein AM at a concentration of $4 \mu \mathrm{M}$ along with Propidium lodide (PI) at a concentration $20 \mu \mathrm{M}$ were added at a total volume of $1 \mathrm{ml}$. The time of incubation was 30 minutes in the dark and care was taken to ensure that the labelling dyes were not overly exposed to light. The scaffolds were washed with PBS twice and then removed and placed in $50 \mathrm{~mm}$ individual wells and then immersed in PBS. The scaffolds were then analysed using an Olympus BX51 confocal microscope. The software used was Lasersharp 2000 
and image stacks of the scaffolds were generated. The outputs of the image stacks were merged and then projected into 3D using ImageJ.

\subsection{Immunostaining for HESMCs}

The scaffolds (10 rpm, $3000 \mathrm{rpm}$ and $5000 \mathrm{rpm}$ and the commercial SIS) were prepared as described previously and placed in identical arrangements using 24-well plates and CellCrown inserts. HESMC's were seeded at a density of 100,000 cells $/ \mathrm{cm}^{2}$. The cells were cultured for 1 day, 4 Days, 7 days and 10 days. The samples were then fixed with 4\% paraformaldehyde. Immunocytochemistry (ICC) staining was then carried out for smooth muscle actin (SMA). After protein blocking by Ultra Vision Block (Lab Vision Corp., Fremont, CA, USA) for 10 min, the primary SMA antibody (Abcam, Cambridge, UK) was applied for $30 \mathrm{~min}$ at a concentration of $5 \mathrm{mg} / \mathrm{ml}$ diluted in antibody diluent (Dako, Glostrup, Denmark) at room temperature in a humidified chamber. The appropriate avidin-biotin complex was applied, with biotinylated goat anti-polyvalent (Abcam, Cambridge, UK) for $10 \mathrm{~min}$, followed by streptavidin peroxidase (Sigma) for a further $10 \mathrm{~min}$.

\subsection{VEGF Incorporation and Release}

Scaffold samples were cut to dimensions of $6 \mathrm{~mm} \times 6 \mathrm{~mm}$ as used in previous analysis studies. A quantity of $10 \mu \mathrm{l}-$ 500ng/scaffold VEGF (Human Recombinant VEGF, Invitrogen, Camarillo, CA, USA) was added to a solution of $0.1 \%$ Bovine Serum Albumin (Sigma) solution previously filtered through a $0.22 \mu \mathrm{m}$ pore sterile filter (Millex ${ }^{\circledR} \mathrm{GP}$, Merck Millipore Ltd. Tullagreen, Co. Cork, Ireland). Under sterile conditions the solution was added in very small increments of $15 \mu \mathrm{l}$ to each sample up to the required $500 \mathrm{ng}$ of VEGF per sample. Following each addition of $15 \mu$ the solution was allowed to dry prior to the addition of the next $15 \mu \mathrm{l}$.

The VEGF was added to samples from 3 scaffold groups: 10, 3000 and $5000 \mathrm{rpm}$. These were tested with and without VEGF alongside a commercial SIS. The samples were placed in $15 \mathrm{ml}$ falcon tubes, immersed in $3 \mathrm{ml}$ of a solution of PBS with $1 \%$ Penicillin-Streptomycin, and placed on an orbital shaker (Stuart Model SSM1) and incubated at $37^{\circ} \mathrm{C}$ for 14 days. At days 1,7 and 14 , aliquots of $150 \mu \mathrm{l}$ were taken and frozen at $-80^{\circ} \mathrm{C}$. The VEGF was quantified using a VEGF Human ELISA Kit (Invitrogen, Camarillo, CA, USA). The aliquots were diluted by a factor of 100 to bring the concentration of VEGF into the range of the specific kit (kit sensitivity being 0 to $1500 \mathrm{pg} \mathrm{mL}^{-1} \mathrm{Hu}$ VEGF) and measured using an absorbance plate reader (M200, Tecan, Männedorf / Switzerland). The values were obtained through the use of a standard curve.

\subsection{Measurement of scaffold angiogenic properties}

VEGF loaded scaffolds were prepared as described previously, and samples were cut to dimensions $6 \mathrm{~mm} \times 6 \mathrm{~mm}$. The VEGF used was recombinant human VEGF (Sf21-derived, 293-VE, R\&D Systems, Inc., and Minneapolis, USA). All samples were sterilised by ethylene oxide at $37^{\circ} \mathrm{C}$ (Person EO50, Person Medical Co., Ltd., Gunpo-si, and Gyeounggi-do, South Korea). For all scaffold groups $n=5$.

The method carried out was a modification of one developed by Storgard [26] The eggs were incubated with rotation for 12-days. Light was then used to identify the air pockets and $2 \mathrm{ml}$ of albumin removed using a needle following an 
incision in the shell under sterile conditions. The incisions were sealed and the eggs were returned to an incubator for a further $24 \mathrm{~h}$. With now enlarged air pockets, the incisions were expanded and the scaffolds were placed on the CAM using a pair of sterile forceps. The eggs were returned to an incubator for 7 and 14 days.

Following incubation $10-15 \mathrm{ml}$ of $10 \%$ formalin (in PBS) was injected into the vital part of the eggs and these were then left at room temperature for $24 \mathrm{~h}$. The egg contents were then discarded and the shells with the intact CAM's were preserved. High resolution digital images of the CAM's were analysed using the Angioquant analysis software [27]. The software automates the identification of vessel complexes and produces a vessel "skeleton" from which the calculations are derived. The calculations are carried out using a stepwise imaging processing methodology involving threshold, segmentation and quantification.

\subsection{Surgical Implantation of Scaffolds}

This study was approved by and carried out to the standards of Dankook University Institutional Animal Care and Use Committee, South Korea. The study was carried out using twenty two 10-week-old male Sprague-Dawley rats. The rats were kept in a temperature $\left(20-24^{\circ} \mathrm{C}\right)$ and humidity $(30-70 \%)$ controlled environment, with $12 \mathrm{~h}$ light and dark cycles, and provided with standard pellet foods and water ad libitum. Prior to implantation each rat received general anaesthesia via an intramuscular injection of ketamine at $80 \mathrm{mg} / \mathrm{kg}$ body weight and xylazine at $10 \mathrm{mg} / \mathrm{kg}$ body weight. All surgical procedure was performed under rigorous sterile conditions and within a controlled sterile environment.

The scaffold samples were prepared and loaded with VEGF as described for the CAM assay. For the implantation, the rats were shaved, a surface wash of iodine solution applied and then a centrally located $2 \mathrm{~cm}$ incision was cut along the back of the animal using a scalpel and mayo scissors. Four separate subcutaneous pouches were created per animal in the region of animal's limbs and a single scaffold samples placed within each. In animals which received VEGF samples only two pouches were created. The incision was sutured using monofilament 4-0 absorbable suture material (Prolene). The animals were then returned to the conditions mentioned above. The animals were later sacrificed at 2 and 4 weeks post implantation.

\subsection{Histological Observation}

The in vivo tissue samples were harvested and then fixed in $10 \%$ neutral buffered saline for $24 \mathrm{~h}$, then immersed in the graded ethanol series for dehydration, and embedded in paraffin. Histological sections were cut at a $5 \mu \mathrm{m}$ thickness from the paraffin blocks using a rotary microtome (Leica RM2245, Leica Biosystems, Germany) and were then stained with haematoxylin and eosin (H\&E). The stained slides which were digitally scanned using a digital slide scanner (Nanozoomer, Hamamatsu, Hamamatsu Photonics K.K., Shizuoka, Japan). Image analysis was carried out using ImageJ. The type of cells which infiltrated were also analysed; three cell types were chosen for analysis, including lymphocytes, foreign-body giant cells (FBGC) and monocytes (Figure 9f, g, h). The cells were counted from sectional images of the scaffolds and the surrounding connective tissues. 


\subsection{Statistical Analysis}

Normal distribution statistical testing was carried out using a 2-tailed student's t-test to a 95\% confidence interval. In the case of the mechanical testing, basic statistical assessment of the data showed that it did not fit normal distribution models and therefore non-parametric statistical analysis methods were used. The group sample data was compared for significant differences using Kruskal-Wallis non-parametric analysis which indicates the presence of significant statistical differences between individual groups. Further analysis was also carried out by post hoc pairwise analysis (Dunnett's Test). All statistical significance tests were done using SPSS versions 20 or 21 (IBM Corporation). 


\section{Results}

\subsection{Alignment of Electrospun Fibres and Scaffold Porosity}

Scaffolds were produced at different rotation speeds (10, 3000 and $5000 \mathrm{rpm})$ and the alignment of electrospun PLGA fibres produced was analysed As the rotation speed increased it was observed that the alignment of the fibres also increased, as shown in the SEM images and orientation distribution graphs (Figure 2). It was also observed that while the alignment varied, the speed of rotation had little effect on the thickness of the fibres. The thickness of the electrospun layers ranged from 11 to $36 \mu \mathrm{m}$ (Figure 3a) with the largest value for the $10 \mathrm{rpm}$ sample group. Care was taken to keep the thickness of the electrospun-fibre layer at a level below which the electrospinning process become affected. The porosity of the electrospun fibres was measured by mercury intrusion (Figure 3b). All the fibre scaffolds showed high porosity levels, and a higher porosity was obtained at a lower rotation speed; $91 \%$ at $10 \mathrm{rpm}>88 \%$ at $3000 \mathrm{rpm}>83 \%$ at $5000 \mathrm{rpm}$. A relationship between the degree of alignment and the porosity was also assessed (Figure 3c). Results showed the degree of alignment correlated inversely with the porosity. Overall, as the rotation speed increased there was an increase in fibre alignment and a reduction in porosity.

As provided in the supplementary data (supplementary figures 7-9), the randomly organised 10 rpm fibre scaffolds showed a normal distribution of pore diameter centred at the $100-150 \mu \mathrm{m}$ range. On the other hand, the $3000 \mathrm{rpm}$ samples showed the distribution was separated into 2 peaks with a higher peak at $5-7.5 \mu \mathrm{m}$, which led to a large fraction of small diameter pores with a simultaneous reduction in large diameter pores. This trend of 2 peaks was also shown in the 5000 rpm samples, however, the fraction of large pores was slightly increased.

Figure 2 Results of SEM images analysis (A)-(C) SEM images of the PLGA fibres at mandrel rotational speeds of 10,3000 \& 5000 rpm (D)-(F) PLGA fibre orientation at 10,3000, 5000 rpm in terms of the percentage of fibres in each of 3 groups spanning 0 - 90 from the mandrel axis of rotation $(n=10-20)$. The error bars are standard deviation. $(G)$ - (I) Frequency histograms for the fibre diameter $(\mu \mathrm{m})$ of the PLGA fibres produced at 10,3000, $5000 \mathrm{rpm}$. The histograms are overlaid with an ideal normal distribution and the mean values $(n=50+)$.

Figure 3 (a) The average thickness of the PLGA electrospun layers for the three alignment groups $(n=15+)$. The error bars are standard deviation. Important values differing significantly from each other are denoted by *; $p<0.05$. (b) Graph representing the porosity of the hybrid scaffolds produced using mercury intrusion porometry $(n=4-6)$. The errors are bars are standard deviation. (c) Graph illustrating the potential trend between fibre alignment and the porosity of the hybrid scaffold groups.

\subsection{Stability of SIS/Fibre scaffold layers}

To optimize the attachment of PLGA fibres to SIS, different conditions were used; varying the diameter of the SIS, electrospinning onto whether the SIS was wet or dry, or insertion of a thin fibre layer by pre-spinning. The tube size and thickness of SIS were shown to be important in determining the attachment (scaffolds images are presented in Figure 4). The percentage of samples which remained attached in an aqueous medium was recorded for the different sample groups (Figure 5). A thinner SIS tube (19 mm diameter, used in the earlier studies; denoted as "Early SIS") led to poor attachment of electrospun fibres for both dry and wet SIS conditions. However, when the SIS tube size was increased (25.4 mm diameter) the fibre attachment was significantly improved. A further slight improvement was also achieved by inserting a thin layer of random-oriented fibres (denoted as "PLGA/SIS + 2 min pre-spin"). 
Figure 4 Images showing (a) the two different sizes of tubing used for processing. A - Working method (2.54 cm diameter) and B Earlier method $(1.9 \mathrm{~cm}$ diameter). The change in diameter resulted in improving the surface smoothness of the SIS due to the stretching effect by the larger tube. (b) The smooth and regular shape of the final SIS scaffold (c) final hybrid scaffold (5000 rpm sample)

Figure 5 The percentage of samples where the SIS and the electrospun layers remained attached. Groups shown include "PLGA/Early SIS: Dry" and "PLGA/Early SIS: Wet" $(n=24)$ using the earlier method with the smaller diameter. The remaining 4 groups all used the standard SIS used in all other work $(n=24)$. Important values differing significantly from each other are denoted by *; $p<05$. $\mathbf{x}$ : The wet SIS group is excluded from statistical analysis due to the practical difficulties in achieving attachment

\subsection{Mechanical Analysis}

The tensile mechanical testing of the scaffolds was carried out in both longitudinal and circumferential direction, as shown in Figure 6. In the longitudinal testing (Figures 6a-c), both the unaligned (10 rpm) and aligned (5000 rpm) scaffolds showed significantly higher modulus values than the control SIS. The yield strength of all the scaffolds was also significantly higher than that of control SIS. The failure strain of scaffolds, particularly for the case of unaligned and partially-aligned (10 and $3000 \mathrm{rpm}$ ), was significantly higher than that of SIS control. Collectively, all the scaffolds did not show significant differences between each other, but showed mechanical improvement (modulus, yield strength, and failure strain) over the control SIS.

In the circumferential testing (Figures 6d-f), the modulus value of the scaffolds were significantly higher than that of SIS control, and the values increased substantially with the increasing degree of alignment; as a result, the modulus for the 5000 rpm scaffold compared to control was more than 10 times higher (40 MPa vs. $3 \mathrm{MPa}$ ). The yield strength of the scaffolds also behaved in a similar manner to the modulus; the $5000 \mathrm{rpm}$ scaffold improved the strength of the SIS control (SIS without fibres) by more than 5 times (1.7 MPa vs. $8 \mathrm{MPa}$ ). On the other hand, the failure strain did not appear to show any trend with all groups showing comparable levels. To summarise the circumferential testing results, the scaffold groups all showed significant improvement over the control SIS in modulus and yield strength, with a trend related to the fibre alignment. However, the exception was the failure strain where all samples showed similar values.

Figure 6 (a) - (c) Mechanical testing data from the longitudinal tensile testing of the hybrid scaffold groups: (a) Elastic modulus values (MPa) $(n=12)$ (b) Yield stress values (MPa) $(n=12)$ (c) Failure strain values (\%) $(n=12)$. (d) - (f) Mechanical testing data from the circumferential tensile testing of the hybrid scaffold groups: (d) Elastic modulus values (MPa) $(n=8)$ (e) Yield stress values (MPa) $(n$ $=8)(\mathrm{f})$ Failure strain values $(\%)(n=8)$. Error bars shown are the standard error. Statistical analysis was done non-parametrically and discussed in the results section 


\subsection{Human Oesophagus Smooth Muscle Cell Response}

The scaffold groups were cultured with HESMCs in a bioreactor and the expression of smooth muscle actin (SMA), was examined. The immunohistochemical staining of SMA demonstrated high expression levels of SMA signals for all the scaffold groups at all culture times (Figure 7).

The metabolic activity analysed by the MTS assay was used to evaluate the biocompatibility of the hybrid scaffolds (Figure. 7b). The MTS level increased with culture time for all the scaffold groups as well as SIS alone and also the commercial (control) SIS. The $3000 \mathrm{rpm}$ scaffold showed a higher MTS level than the other sample groups at days 1 and 7 .

Live-Dead assay indicated the live cell fraction was mainly over $80 \%$ for all the hybrid scaffolds up to the test period of 14 days; however, control SIS groups showed a reduction in live cell fraction to $50-55 \%$ at day 14 (Figure $7 d$ ). The representative 3D projection images of $10 \mathrm{rpm}$ scaffold are shown in Figure $7 \mathrm{~d}$. There is also some visual evidence for cell alignment for the 5000rpm scaffolds (See Figure 8)

Figure 7(a) Histological images demonstrating positive IHC staining for smooth muscle actin (SMA) from HESMC's cultured on the commercial control SIS in addition to the hybrid scaffold groups $(10,3000$ and $5000 \mathrm{rpm}$ ) at Days 1, 4, 7 and 10. (b) Graph representing the normalised levels of metabolic activity of HESMC's cultured on the hybrid scaffold groups. The values are expressed as a percentage of the control (in this case the HESMC's cultured on TCP). (c) Graph representing the percentage of live cells observed by performing a Live-Dead assay on HESMC's cultured on the scaffold groups. The values were obtained cell counts from confocal microscopy images and image stacks ( $n$ is between 9 and 20 images or image stacks). (d) Series of confocal images $(10 \mathrm{rpm})$ showing the 3D projections of a stack of confocal images for the purpose of live-dead analysis

Figure 8 Confocal images demonstrating different cell orienations: (a) random - $10 \mathrm{rpm}$ at Day 7 and (b) aligned cells - 5000 rpm at Day 14

\subsection{In vivo Tissue Compatibility and Cell Infiltration}

The hybrid scaffolds were implanted subcutaneously for 4 weeks and the tissue samples were analysed for in vivo biocompatibility. VEGF-incorporated scaffold groups were also used for the comparison. The degree of in vivo cellular infiltration was first analysed. For porous and degradable scaffolds to function, host cells (immune and other tissue cells) were required to infiltrate into the scaffold. Generally, the pure SIS control was readily infiltrated (Figure 9a), but the commercial SIS scaffold was poorly infiltrated (Figure 9b). The hybrid scaffold groups were variable in the infiltration depending on the scaffold types (Figure 9c,d). When quantified by infiltration score (Figure 9e), the SIS control showed the highest infiltration, whilst the comercial SIS and the random fibre orientation fibre group (10 rpm) were the lowest. Moreover, an increase in fibre aligment enhanced the infiltration; $5000 \mathrm{rpm}>3000 \mathrm{rpm}>10$ rpm. The VEGF incorporated within hybrid scaffolds did not significantly affect the cellular infiltration. It was also observed that the surrounding connective tissue was predominantly in the region where the blood vessels were located compared to the capsule region where the cell numbers were very low. The effects of VEGF on the in vivo blood vessel formation are detailed in the following section.

With respect to the ratio of the types of cells which were present, the first observation made was that there was a lack of FBGCs with the control SIS scaffolds compared to other groups and this was easily identifiable through 
observation at low magnification (Figure 8). Beyond this, all the hybrid scaffolds all the groups performed very similarly. The lymphocyte numbers for the hybrid groups also fell from 2 to 4 weeks. Also in all sample groups there was a high number of plasma cells (involved in antibody production). Also see supplementary figure 10.

Figure 9 Histological images (H\&E) illustrating general observations of the in vivo samples (a) Typical features (3000rpm - 4 weeks) demonstrating the (A1) underlying muscular tissue (A2) surrounding granulation tissue (A3) encapsulated scaffold (b) Pure SIS sample (Day 7), B1-Pink stained collagen-based SIS which is well infiltrated, B2- Boundary between the capsule and the underlying muscle tissue (c) PLGA-SIS 5000 rpm (Day7), C1-Well infiltrated PLGA Fibres, C2- Well infiltrated SIS (c) PLGASIS 5000 rpm (Day7), (d) D1-Poorly infiltrated electrospun fibres, and D2- Poorly infiltrated SIS (e) Graph of the mean cellular infiltration score. The images were scored from 0-4 with 0 being poor infiltration and 4 being highly infiltrated $(n=4$ and the error bars are standard deviation). Graphs ( $\mathrm{g}-\mathrm{h})$ : The average number of cells per $\mathrm{mm} 2$ of histologically visible scaffold for the following the cell types: (f) Lymphocytes (g) Monocytes (h) FBGC's ( $n=3$ and the error bars are the standard deviation). Graphs (i-k): The average number of cells per $\mathrm{mm} 2$ of histologically visible sounding connective tissue for the following the cell type (d) Lymphocytes (e) Monocytes (f) FBGC's ( $n=3$ and the error bars are the standard deviation).

\subsection{Effects of VEGF Release from scaffols and Angiogenesis in CAM Model and Subcutaneous Tissue}

The VEGF release assay showed that VEGF was present at detectable levels in all samples (Figure 10a). The VEGF levels did not vary significantly between the samples or over time. It was also noted that a trace amount of VEGF was detected from the commercial control at day 7 . The total quantity of VEGF released by the scaffolds was at most found to be slightly over $100 \mathrm{ng}$; this contrasts with the initial quantity of VEGF of $500 \mathrm{ng}$ per sample. The effect of VEGF release on the angiogenesis was then assayed in a CAM model. Using Angioquant software, the mean length of blood vessel and the number of junctions within identified vessel complexes on the CAM were analysed for each scaffold group. The average blood vessel length in all the VEGF-free scaffold groups did not differ significantly from the control (Figure 10b). On the contrary, all the scaffold groups with VEGF showed significantly higher blood vessel length than the control. When it came to the difference between identical scaffold groups with and without VEGF, all had larger values in the presence of VEGF. The number of junctions was also characterized (Figure 10c). All the samples containing VEGF showed higher values than the control and VEGFfree samples.

The density of blood vessels formed in vitro within the scaffolds as well as in the surrounding tissues was then analysed (Figures 10d and e and supplementary figure 11). All the sample groups showed higher blood vessel density in the surrounding connective tissue than within the scaffolds. When compared between groups, blood vessel density was higher in the VEGF- containing scaffolds than in the VEGF-free scaffolds for both within the scaffold region and in the surrounding tissue. 
Figure 10 (a) Graph representing the concentration of VEGF in solution following hybrid scaffold immersion at day intervals of 1 , 7 and 14, along with a commercial SIS and a control (in this instance $10 \mathrm{rpm}$ with no VEGF). The "+" denotes a trace amount of VEGF detected in the commercial VEGF on day $7(n=4$ and the error bars are standard deviation). (b) The mean length of vessel complex on the CAM for the different scaffold groups as identified by quantification software. The sample groups shown are (with $n$ number in brackets) : Control (24), $10 \mathrm{rpm}$ (21), $3000 \mathrm{rpm}$ (15), $5000 \mathrm{rpm}$ (21), $10 \mathrm{rpm}+$ VEGF (20), $3000 \mathrm{rpm}+$ VEGF (26), and $5000 \mathrm{rpm}+$ VEGF (25). (c) The mean number junctions found per vessel complex for the different scaffold groups on the CAM as identified by quantification software. The sample groups shown are: Control (24), $10 \mathrm{rpm}$ (21), $3000 \mathrm{rpm}$ (15), $5000 \mathrm{rpm}$ (21), $10 \mathrm{rpm}+$ VEGF (20), $3000 \mathrm{rpm}+$ VEGF (26), and $5000 \mathrm{rpm}+$ VEGF (25). All error bars are the standard deviation. Important values differing significantly from each other are denoted by $; p<0.05$. The average number of histologically visible blood vessels per $\mathrm{mm}^{2}$ of tissue based on histological analysis $(n=4$ for the number of sample images and the error bars are the standard deviation). The bars are alternatively shaded between groups at weeks 2 and 4 . 


\section{Discussion}

\subsection{Electrospun Fibre Stability}

The problem about efficiency of attachment between the layers of ECM material and electrospun fibres has been addressed previously depending on the material and conditions of electrospinning. Horst et al. found it to be an issue when using sheets of decellularised urinary bladder matrix (UBM) which was to be coated with electrospun PLGA fibres. To achieve any significant attachment it was required that the UBM be constantly rehydrated during the electrospinning [19]. When a similar approach was attempted here it was found not to work. However the shape of the SIS and the rotation of the mandrel are likely to have prevented the method used here from working as the constant rehydration caused the SIS to sag on the mandrel and thus creating a mobile and less stable surface onto which the electrospun fibres could attach. It contrasts significantly with a flat hydrated matrix in which case the stability of the surface is likely increased with hydration as the surface tension of the material to the flat electrode beneath removes any "wrinkling" in the material that observed in testing however a flat material has its own limitations like specimen curvature prior to testing.

The challenge here was to improve the attachment of the electrospun fibres to the SIS while maintaining the cylindrical shape. It was observed that the early forms of the SIS had a very wrinkled and uneven surface. The reason was due to the disparity in the diameter of the SIS and the diameter of the porous polyethylene tubing onto which it was placed prior to vacuum drying and resulted in "slack" along the SIS lengths becoming compressed and drying in a variety of peaks and troughs. In turn, this not only provided a rough surface onto which to electrospin but the topography also allowed for spans of electrospun fibres having no surface contact with the SIS. Increasing the diameter of the porous tubing resulted in the SIS being elastically stretched, and while a more difficult process, this resulted in a smoother surface. The surface was further made smooth by the compression of the bag during vacuum drying phase. This is the opposite of the circumstance with the previous iteration of the SIS where the vacuum exacerbated the irregularities due to the excess present. Consequently when the new surface was electrospun on to, it showed a significant improvement in the levels of fibre attachment.

The reason for the increase in the adhesion level is likely due to the increased interaction between the SIS and the electrospun fibres. It has been shown that adhesive forces exist between fibres and substrates $[28,29]$. Examples described include the fibrillary hairs (or setae) found on the Gekko's foot, and the principle mechanical force is believed to be van der Waals (vdW). This is also the case for adhesion that has been described for fibre-fibre adhesion [30]. Consequently, by increasing the smoothness of the SIS surface it was possible to increase the contact surface area and therefore the level of adhesion. It was noted that inadequate drying resulted in incomplete drying of fibres which has the result of causing some increased attachment (results shown in Figure 5).

\subsection{Hybrid Scaffold Evaluation}

Following successful adhesion and hybrid scaffold creation, one of the key variables which emerged was the alignment of the PLGA fibres. Altering the fibre alignment resulted in a number of changes to the properties of the 
scaffold. It was observed that the fibre diameter was consistent in all scaffold groups. This was consistent with other studies where the rotational speed of a mandrel has not been shown to affect the fibre diameter [31]. However this was important to ascertain as there are examples where a high enough rotational speed can cause a spooling effect where the fibres are thinned by the process [32]. This may contribute to understanding the reason behind the fact that levels of attachment were also found to be consistent across the hybrid groups.

With the mechanical properties, the alignment of the fibres did create some difference which is discussed in detail below, though there were some general observations which could be made. Firstly, in the circumferential direction the failure strain was found to be well above $50 \%$ in all groups, which is important as this is the degree of expansion in the human oesophagus during swallowing. In the longitudinal direction all the scaffolds had failure strains above $70 \%$, which was again important, as this is around the values quoted for the ultimate strain of the human oesophagus in the longitudinal direction [33]. In addition to this, none of the hybrid groups performed worse than the control (unaltered SIS) in any of the testing methods used. This highlights that in addition to the fibres acting as an in vivo support structure, they also offer an improvement in the mechanical properties over the control SIS. Morover, as well as the physical properties of the scaffolds, the biocompatibility is also of paramount importance. Biocompatibility was first evaluated my means of metabolic activity and it was found that all scaffold groups performed as well as the control and the commercial SIS. This was an important result particularly when compared to a commercial SIS which is currently in use on the market. The Live-Dead assay further confirmed that no major differences were detected between any of the hybrid scaffold group, in addition to both the commercial SIS control and the control (cells on tissue culture well plate). The scaffolds were also cultured and tested for the presence of smooth muscle actin (SMA), a vital component of smooth muscle cell contractility. All the scaffold were shown to the not impair production of SMA by the HESMC's during in vitro evaluation.

Several observations were made from the live-dead assay. Firstly that there was no difference detected between the hybrid scaffold groups which were tested. Secondly, that the entire hybrid scaffold groups showed a similar level of inferred biocompatibility to the control TCP and the commercial SIS. A final observation was that using the confocal it was easy to identify the alignment of the cells along the orientation of the fibres.

When the scaffolds were implanted in vivo the results were a little more complex to evaluate. All samples elicited an immune inflammatory reaction around the scaffold and provoked the formation of a thin layer of granulation connective tissue surrounding the scaffold. Close inspection revealed differences in the immune reactions even though an approximation of the cell numbers showed very little differences between groups.

The first clear observation is that there was cellular infiltration and encapsulation of all samples as expected of any foreign object being placed subcutaneously. Upon implantation there is an immediate phase of acute inflammation, which can last up to a week, and which is characterised in its very earliest phases by neutrophils, mast cell degranulation releasing histamine (along with the critical Interleukins $3 \& 4$ ) and with fibrinogen adsorption on the implanted biomaterial surface [34].This is followed by a chronic phase of inflammation which occurs for a longer 
and more variable period. The chronic inflammatory phase is considered to be defined by the presence of mononuclear cells, i.e. monocytes and lymphocytes, at the site of implantation and is the phase that concerns this work (weeks 2 and 4). When chronic inflammation is used to describe the foreign body reaction it is often described as when monocytes, macrophages, and foreign body giant cells are all present at the biomaterial interface [35]. The formation of FBGC's is an important indicator of the foreign body reaction and have been shown to exhibit an antigenic phenotype similar to both monocytes and macrophages reflecting their formation from the fusion of monocyte-derived macrophages [36]. It has also been shown that lymphocytes also have a role to play in the foreign body reaction. Adherent lymphocytes have been shown to predominately associate with macrophages and FBGC's rather than the surface of the biomaterial itself [37].

Histological evaluation of the samples here showed that the control SIS did not cause a foreign body reaction as shown by the lack of FBGC's and this is quite an important result in that a largely collagenous xenogeneic decellularised tissue was shown to function with a minimal inflammatory response in vivo. The commercial SIS undoubtedly had all the early indications of a foreign body reaction at 2 weeks however by 4 weeks this had resolved itself and the numbers were very low.

For the hybrid scaffolds, all groups showed very similar results, had large numbers of FBGC's. Macrophages are only capable of phagocytosing very small particles $(<5 \mu \mathrm{m})$ and the presence of larger particle sizes $(>10 \mu \mathrm{m})$ or large fibres, can induce the formation of foreign body giant cells [35]. In the case of hybrid scaffolds the PLGA was in the form of nanofibers and so with very high surface areas and these effects would be prevalent and could contribute greatly to the foreign body reaction. The lymphocyte numbers also fell from week 2 to 4 (the exception being for the $3000 \mathrm{rpm}$ sample however there was high variability in this group with at least one sample showing an almost unique proportion of cells potentially skewing the mean value). This appears to indicate that by 4 weeks the roles of the lymphocytes in any on-going inflammatory processes was quite reduced. What can also be said is that on visual assessment of the images, that in all samples there was a high number of plasma cells (involved in antibody production). These are successor cells to lymphocytes and could also account for the reduction in number. Analysis of all sample groups together, it is clear that that the presence of the PLGA electrospun layer is largely the cause of the foreign body reaction to the PLGA-SIS scaffold. What should be emphasized here is that although this was not an aggressive response and it compared well with other types of PLGA scaffolds which have been implanted in vivo $[38,39]$

With these inflammatory responses in effect it is important to note that the PLGA scaffold is intended to be a temporary feature. This falls in line with the main objectives of this work in that the PLGA is required to maintain its mechanical integrity for 2-4 weeks and then begin to be degraded as the scaffold gives way to regenerated oesophageal tissue. Consequently for a scaffold such as this to succeed the degradation process is an essential part of the function of the implant. That said, the scaffold should degrade consistently throughout its structure and 
large sections of the scaffold which are not infiltrated risk becoming isolated and this will be discussed later. To evaluate the degradation profile longer term in vivo experiments may be investigated for future researches.

\subsection{Effects of Fibre Alignment}

The mechanical properties were not found to be affected by the alignment of the fibres with the exception of the highly aligned scaffolds, which greatly increased toughness was observed in the circumferential direction. This result shows a parallel outcomes with other studies. [21, 40]. This does demonstrate some advantage over the less aligned groups as the force of the intravenous bolus would be directed mainly in the circumferential direction. It is well known that for a scaffold to support cell viability in addition to tissue growth and development it must have sufficient porosity to allow for transport of nutrients and the removal of waste products [41]. In regards to porosity, nanofibre electrospun scaffold have been described as often being limited [42]. It was not necessarily observed here as all the scaffold groups achieved porosity levels higher than $80 \%$ and some difference could be attributed to the use of a rotating mandrel. The porosity data also showed that the level of the porosity decreased with the alignment which has also been observed in other work [31]. This difference is also reflected in the differences in the thickness of the scaffolds between the $10 \mathrm{rpm}$ (unaligned) and the other two groups. Visual inspection also confirmed this difference as the unaligned scaffolds did appear slightly thicker. The finding is significant because the groups with more aligned fibres could potentially be made at the same thickness but using more fibrous material, and hence having better reinforcement and this is an advantage because there is a limit to the thickness to which the electrospun layer can be made. It has been reported that with an increase in the thickness of the electrospun layer, residual charge accumulation on the deposited fibres interferes with the incoming fibres [22, 43]. This was observed during this work, as this "insulating" effect which caused the fibres to be laterally deflected away from the SIS to the exposed metal mandrel on either side.

Another, potentially significant, aspect about porosity is the pore size distribution. Pore radius size distribution was shown to change when varying the alignment of the fibres. As seen from the sample graphs, the pore distribution skews in both directions as the fibres become more aligned, essentially meaning that a higher proportion of the pore diameters were at the extreme ends of the pore size range. It is important to note that both extremes of pore size have been reported to having in vivo significance. From the relevant literature it is suggested that for scaffolds to function in tissue engineering applications an outer pore size in the range of 50 to $200 \mu \mathrm{m}$ is able to facilitate cell seeding, and transport of waste products and nutrients, and smaller pores in the range of 35 to $70 \mu \mathrm{m}$ are considered important for the promotion of angiogenesis [6, 33, 44, 45].

On histological analysis of the in vivo samples, there was a difference in the level of cellular infiltration between the scaffold groups. The trend here was that the higher the degree of fibre alignment, the higher the levels of cellular infiltration. This is a finding which has been previously reported, where electrospun scaffolds were inserted in vivo and the more aligned fibres demonstrated better cellular infiltration than those with random orientations [47]. The alignment of the fibres have also been described as facilitating cell movement within scaffolds that is shown with 
periodontal cells in this instance [46]. That being said, it has also been demonstrated that fibre alignment does not affect integration [47].

In this study, it was found aligned fibres produced better cellular infiltration in vivo which is a positive aspect for trasplantation applications in tissue engineering. The cause for this is likely to be due to combinations of the factors described here including pore size distribution and facilitated cell movement within scaffolds by fibre alignment. An additional observation was that on in vitro confocal analysis the cells were found in be present in "seams".

One of the key goals of fibre alignment was to facilitate aligned smooth muscle cell growth and was confirmed in this work with confocal imaging. The use of aligned electrospun fibres with contact guidance dictating the orientation of human smooth muscle cells has become a relatively common one in the field of arterial tissue engineering [20, $40,48]$. In one case, human umbilical artery smooth muscle cells were cultured onto aligned PCL fibres and here it was found that and not only was the orientation and the morphology of the cells influenced by the fibre alignment, but that also the distribution and the organisation of the cytoskeletal proteins within the SMC's were also found to be parallel to the direction of the fibres [22]. In addition to orientation, smooth muscle cells cultured in this way have also been shown to express the genes for the contractile proteins (calponin, smooth muscle a-actin (SMA), SM22, and smoothelin) [49]. Furthermore, it has also been observed that following cellular orientation there is also fibrous extracellular matrix formation which also follows the same alignment [50]. These phenomena all contribute to potentially addressing the issue prevalent in a wide variety of regenerative applications where the tissues produced are only in the form of disorganised fibres.

\subsection{Effects of VEGF delivery on angiogenesis}

Due to its water-soluble nature it cannot be readily incorporated into electrospun fibres however by using adsorbed VEGF it has been successfully applied onto the scaffold prior to in vivo implantation [51-55]. The results showed the release profile was largely identical for all the scaffold groups tested.

In a study also using VEGF adsorption, it was found that there was an initial burst release after 1 day, as observed here, in addition to a small and sustained release over the course of days 2-5 [51]. In another study there was also a burst release at day one followed by a slow decrease to virtually nothing by day 7 [54]. Even though a longer time-scale was used here, unfortunately it cannot confirm the rate of this gradual decline though the overall trend remains the same. The quantity of VEGF was also found to be between $60-110 \mathrm{ng}$, which could be considered quite low given the initial quantity added was $500 \mathrm{ng}$ per sample.

It is well established that VEGF can affect the CAM [56-58]. The main conclusion of the CAM assay is that VEGF was successfully incorporated into the scaffold and the average length of the complexes was statistically higher for the VEGF scaffold groups versus the control which was not the case for the non-VEGF scaffold groups. It was also demonstrated that when the data from all the VEGF samples were combined and then these were compared to the combined non-VEGF samples, there was a statistically significant difference between the two sets in terms of 
the mean number of junctions. In conclusion it was demonstrated that in an in vivo model the scaffolds and the VEGF can have a direct effect by increasing angiogenesis.

When scaffolds were implanted in an animal model the results showed the incorporation of VEGF caused a statistically significant increases in the blood vessel density in the surrounding granulation tissue. This is a result which has been observed previously, including increased microvessel density with VEGF added to a PLGA sub dermal implant for bone tissue applications [59].

It is also important to state that VEGF is an angiogenic factor that has only been used with limited success. There is a large body of work that shows that VEGF, when used in isolation can induce vessels which are immature and leaky, giving rise to local oedema and inefficient tissue perfusion [60]. Consequently this represents a limitation of the work; however the VEGF loading was also an important method by which quantifiable data could be obtained post in vivo implantation to demonstrate that the scaffolds had the potential for drug delivery.In addition, organised and functional scaffold re-population in vitro before transplantation maximizes both the ingrowth of neighbouring host cells and angiogenesis[61] .

In general due its complex structure, the oesophageal tissue have several challenges to overceom priorto achieving clinically successful grafting. Because of the multi-layered nature of oesophageus it is promising to utulise tissue engineering strategies to mimic and form all structural compartments for its recovery.

\section{5 - Conclusion}

In conclusion the electrospun fibrous polymer layer onto tubular SIS was developed and that attachment was achievable between the two layers so they could be treated as a single structure. The presented approach was based on a combined structure of ECM and biodegradable PLGA and it appears to be a successful route for oesophageal tissue engineering. The scaffolds were shown to have good mechanical properties, biocompatibility, and porous morphology all of which made them suitable scaffold materials. It was further concluded that of the three groups, the group with the highly-aligned fibres $(5000 \mathrm{rpm})$ was the most promising one for the purposes of oesophageal tissue engineering. The alignment of PLGA fibers increased with rotation speeds and the degree of alignment correlated inversely with the porosity. The PLGA - SIS hybrid scaffold has mechanical improvement over the control SIS. Confirmation of alignment of HESMC's when cultured in vitro, showed higher levels of cellular infiltration when implanted in vivo, and the increased circumferential mechanical toughness, all contributed to this conclusion. The addition of VEGF increased local angiogenesis and so could improve the functionality of the scaffold.

For future research, based on this data, PLGA has been shown to improve the level of response to the PLGA, reducing the time taken for resorption, surface modification and active agents can all be used and other alternative factors can also be used. The stent-like support of the rapidly remodelling ECM, in addition to the inherent mechanical properties of the electrospun fibres, points to the potential of this methodology to be taken forward. 
Furthermore, using fibre alignment can also be used to influence the orientation of cellular growth of muscle cells. Significantly the concept of creating a hybrid material which does not use chemical modification of the component materials and so will not alter their individual properties represents an important approach in this field.

Acknowledgements: This work was supported in part by National Research Foundation (2018K1A4A3A01064257 and 2018R1A2B3003446), Republic of Korea. 


\section{References}

[1] A.J. Holland, D.A. Fitzgerald, Oesophageal atresia and tracheo-oesophageal fistula: current management strategies and complications, Paediatr Respir Rev 11(2) (2010) 100-6; quiz 106-7.

[2] L. Spitz, E.M. Kiely, D.P. Drake, A. Pierro, Long-gap oesophageal atresia, Pediatr. Surg. Int. 11(7) (1996) 462-465.

[3] G. Totonelli, P. Maghsoudlou, F. Georgiades, M. Garriboli, K. Koshy, M. Turmaine, M. Ashworth, N.J. Sebire, A. Pierro, S. Eaton, P. De Coppi, Detergent enzymatic treatment for the development of a natural acellular matrix for oesophageal regeneration, Pediatr. Surg. Int. 29(1) (2013) 87-95.

[4] L. Ludman, L. Spitz, Quality of life after gastric transposition for oesophageal atresia, Journal of pediatric surgery 38(1) (2003) 53-57.

[5] P. Maghsoudlou, S. Eaton, P. De Coppi, Tissue engineering of the esophagus, Seminars in Pediatric Surgery 23(3) (2014) 127-134.

[6] T. Poghosyan, S. Gaujoux, R. Sfeir, J. Larghero, P. Cattan, Bioartificial Oesophagus in the Era of Tissue Engineering, J. Pediatr. Gastroenterol. Nutr. 52 (2011) S16-S17.

[7] A.J. Allman, T.B. McPherson, S.F. Badylak, L.C. Merrill, B. Kallakury, C. Sheehan, R.H. Raeder, D.W. Metzger, Xenogeneic extracellular matrix grafts elicit a TH2-restricted immune response, Transplantation 71(11) (2001) 1631-40.

[8] F.H. Bach, C. Ferran, P. Hechenleitner, W. Mark, N. Koyamada, T. Miyatake, H. Winkler, A. Badrichani, D. Candinas, W.W. Hancock, Accommodation of vascularized xenografts: expression of "protective genes" by donor endothelial cells in a host Th2 cytokine environment, Nature Medicine 3(2) (1997) 196-204.

[9] T.J. Keane, R. Londono, N.J. Turner, S.F. Badylak, Consequences of ineffective decellularization of biologic scaffolds on the host response, Biomaterials 33(6) (2012) 1771-81.

[10] B.N. Brown, J.E. Valentin, A.M. Stewart-Akers, G.P. McCabe, S.F. Badylak, Macrophage phenotype and remodeling outcomes in response to biologic scaffolds with and without a cellular component, Biomaterials. 30(8) (2009) 1482-91. Epub 2009 Jan 1.

[11] S.F. Badylak, D. Taylor, K. Uygun, Whole-Organ Tissue Engineering: Decellularization and Recellularization of Three-Dimensional Matrix Scaffolds, in: M.L. Yarmush, J.S. Duncan, M.L. Gray (Eds.), Annual Review of Biomedical Engineering, Vol 13, Annual Reviews, Palo Alto, 2011, pp. 27-53.

[12] T. Doede, M. Bondartschuk, C. Joerck, E. Schulze, M. Goernig, Unsuccessful Alloplastic Esophageal Replacement With Porcine Small Intestinal Submucosa, Artificial Organs 33(4) (2009) 328-333.

[13] S. Badylak, S. Meurling, M. Chen, A. Spievack, A. Simmons-Byrd, Resorbable bioscaffold for esophageal repair in a dog model, Journal of pediatric surgery 35(7) (2000) 1097-1103.

[14] A. Clough, J. Ball, G.S. Smith, S. Leibman, Porcine Small Intestine Submucosa Matrix (Surgisis) for Esophageal Perforation, The Annals of Thoracic Surgery 91(2) (2011) e99-e100-e99-e100.

[15] O. Syed, N.J. Walters, R.M. Day, H.-W. Kim, J.C. Knowles, Evaluation of decellularization protocols for production of tubular small intestine submucosa scaffolds for use in oesophageal tissue engineering, Acta Biomaterialia 10(12) (2014) 5043-5054.

[16] S. Badylak, M. Kokini, B. Tullius, B. Whitson, Strength over time of a resorbable bioscaffold for body wall repair in a dog model, Journal of Surgical Research 99(2) (2001) 282-287.

[17] I.O. Asencio, S. Mittar, C. Sherborne, A. Raza, F. Claeyssens, S. MacNeil, A methodology for the production of microfabricated electrospun membranes for the creation of new skin regeneration models, J Tissue Eng 9 (2018) 2041731418799851.

[18] M.J. McClure, S.A. Sell, D.G. Simpson, B.H. Walpoth, G.L. Bowlin, A three-layered electrospun matrix to mimic native arterial architecture using polycaprolactone, elastin, and collagen: $A$ preliminary study, Acta Biomaterialia 6(7) (2010) 2422-2433.

[19] M. Horst, S. Madduri, V. Milleret, T. Sulser, R. Gobet, D. Eberli, A bilayered hybrid microfibrous PLGA-Acellular matrix scaffold for hollow organ tissue engineering, Biomaterials 34(5) (2013) 15371545. 
[20] L. Jia, M.P. Prabhakaran, X. Qin, S. Ramakrishna, Guiding the orientation of smooth muscle cells on random and aligned polyurethane/collagen nanofibers, Journal of biomaterials applications (2014).

[21] A. Subramanian, U.M. Krishnan, S. Sethuraman, Fabrication of uniaxially aligned 3D electrospun scaffolds for neural regeneration, Biomedical Materials 6(2) (2011).

[22] Y.B. Zhu, Y. Cao, J. Pan, Y.X. Liu, Macro-Alignment of Electrospun Fibers For Vascular Tissue Engineering, J. Biomed. Mater. Res. Part B 92B(2) (2010) 508-516.

[23] D. Leung, G. Cachianes, W. Kuang, D. Goeddel, N. Ferrara, Vascular endothelial growth factor is a secreted angiogenic mitogen, Science 246(4935) (1989) 1306-1309.

[24] G.C. Lantz, S.F. Badylak, A.C. Coffey, L.A. Geddes, W.E. Blevins, Small intestinal submucosa as a small-diameter arterial graft in the dog, Journal of investigative surgery : the official journal of the Academy of Surgical Research 3(3) (1990) 217-27.

[25] S.F. Badylak, Small intestinal submucosa as a large diameter vascular graft in the dog, The Journal of surgical research 47(1) (1989) 74-80.

[26] C. Storgard, D. Mikolon, D. Stupack, Angiogenesis Assays in the Chick CAM, in: J.-L. Guan (Ed.), Cell Migration, Humana Press2005, pp. 123-136.

[27] A. Niemisto, V. Dunmire, O. Yli-Harja, W. Zhang, I. Shmulevich, Robust quantification of in vitro angiogenesis through image analysis, IEEE Trans. Med. Imaging 24(4) (2005) 549-553.

[28] K. Autumn, Y.A. Liang, S.T. Hsieh, W. Zesch, W.P. Chan, T.W. Kenny, R. Fearing, R.J. Full, Adhesive force of a single gecko foot-hair, Nature 405(6787) (2000) 681-5.

[29] H. Gao, H. Yao, Shape insensitive optimal adhesion of nanoscale fibrillar structures, Proceedings of the National Academy of Sciences of the United States of America 101(21) (2004) 7851-6.

[30] Q. Shi, K.-T. Wan, S.-C. Wong, P. Chen, T.A. Blackledge, Do Electrospun Polymer Fibers Stick?, Langmuir 26(17) (2010) 14188-14193.

[31] C. Ayres, G.L. Bowlin, S.C. Henderson, L. Taylor, J. Shultz, J. Alexander, T.A. Telemeco, D.G. Simpson, Modulation of anisotropy in electrospun tissue-engineering scaffolds: Analysis of fiber alignment by the fast Fourier transform, Biomaterials 27(32) (2006) 5524-34.

[32] F. Yang, R. Murugan, S. Wang, S. Ramakrishna, Electrospinning of nano/micro scale poly(L-lactic acid) aligned fibers and their potential in neural tissue engineering, Biomaterials 26(15) (2005) 26032610.

[33] J.Y. Tan, C.K. Chua, K.F. Leong, K.S. Chian, W.S. Leong, L.P. Tan, Esophageal tissue engineering: An in-depth review on scaffold design, Biotechnology and bioengineering 109(1) (2012) 1-15.

[34] J. Zdolsek, J.W. Eaton, L. Tang, Histamine release and fibrinogen adsorption mediate acute inflammatory responses to biomaterial implants in humans, Journal of translational medicine 5(1) (2007) 31.

[35] J.M. Anderson, A. Rodriguez, D.T. Chang, Foreign body reaction to biomaterials, Seminars in immunology 20(2) (2008) 86-100.

[36] N.A. Athanasou, J. Quinn, Immunophenotypic differences between osteoclasts and macrophage polykaryons: immunohistological distinction and implications for osteoclast ontogeny and function, Journal of Clinical Pathology 43(12) (1990) 997-1003.

[37] M. Macewan, E. Colton, H. Meyerson, W.G. Brodbeck, J.M. Anderson, Lymphocytes and the foreign body response: Lymphocyte enhancement of macrophage adhesion and fusion, Journal of biomedical materials research. Part A 74(2) (2005) 222-229.

[38] K. Blackwood, R. McKean, I. Canton, C. Freeman, K. Franklin, D. Cole, I. Brook, P. Farthing, S. Rimmer, J. Haycock, A. Ryan, S. MacNeil, Development of biodegradable electrospun scaffolds for dermal replacement, Biomaterials 29(21) (2008) 3091-3104.

[39] F. Han, X. Jia, D. Dai, X. Yang, J. Zhao, Y. Zhao, Y. Fan, X. Yuan, Performance of a multilayered smalldiameter vascular scaffold dual-loaded with VEGF and PDGF, Biomaterials 34(30) (2013) 7302-13.

[40] L. Nivison Smith, A. Weiss, Alignment of human vascular smooth muscle cells on parallel electrospun synthetic elastin fibers, Journal of biomedical materials research. Part A 100(1) (2012) 155-161. 
[41] B. Sharma, J.H. Elisseeff, Engineering Structurally Organized Cartilage and Bone Tissues, Annals of biomedical engineering 32(1) (2004) 148-159.

[42] L.T.H. Nguyen, S. Chen, N. Elumalai, M. Prabhakaran, Y. Zong, C. Vijila, S. Allakhverdiev, S. Ramakrishna, Biological, Chemical, and Electronic Applications of Nanofibers, Macromolecular materials and engineering 298(8) (2013) 822-867.

[43] W.E. Teo, S. Ramakrishna, A review on electrospinning design and nanofibre assemblies, Nanotechnology 17(14) (2006) R89-R106.

[44] A. Ritchie, K. Chian, B. Beckstead, B. Ratner, Esophagus: A Tissue Engineering Challenge, in: J.D. Bronzino (Ed.), Tissue engineering and Artificial Organs, CRC Press2006, pp. 54-1-54-19.

[45] P. Kuppan, S. Sethuraman, U.M. Krishnan, Tissue engineering interventions for esophageal disorders - Promises and challenges, Biotechnology Advances 30(6) (2012) 1481-1492.

[46] S. Shang, F. Yang, X. Cheng, X.F. Walboomers, J. Jansen, The Effect of Electrospun Fibre Alignment on the Behaviour of Rat Periodontal Ligament Cells, European cells \& materials 19 (2010) 180-192.

[47] L.C. Ionescu, R.L. Mauck, Porosity and Cell Preseeding Influence Electrospun Scaffold Maturation and Meniscus Integration In Vitro, Tissue engineering. Part A 19(3-4) (2013) 538-547.

[48] E. Bell, Chapter Seventeen - Models as precursors for prosthetic devices, in: R.L.L. Vacanti (Ed.), Principles of Tissue Engineering (Third Edition), Academic Press, Burlington, 2007, pp. 241-250.

[49] S. Rayatpisheh, D.E. Heath, A. Shakouri, P.-O. Rujitanaroj, S.Y. Chew, M.B. Chan-Park, Combining cell sheet technology and electrospun scaffolding for engineered tubular, aligned, and contractile blood vessels, Biomaterials (0) (2013).

[50] J. Glass-Brudzinski, D. Perizzolo, D.M. Brunette, Effects of substratum surface topography on the organization of cells and collagen fibers in collagen gel cultures, Journal of Biomedical Materials Research 61(4) (2002) 608-618.

[51] S. Singh, B.M. Wu, J.C.Y. Dunn, Delivery of VEGF using collagen-coated polycaprolactone scaffolds stimulates angiogenesis, J. Biomed. Mater. Res. Part A 100A(3) (2012) 720-727.

[52] I. Freeman, S. Cohen, The influence of the sequential delivery of angiogenic factors from affinitybinding alginate scaffolds on vascularization, Biomaterials 30(11) (2009) 2122-2131.

[53] E. Wernike, M.O. Montjovent, Y. Liu, D. Wismeijer, E.B. Hunziker, K.A. Siebenrock, W. Hofstetter, F.M. Klenke, Vegf incorporated into calcium phosphate ceramics promotes vascularisation and bone formation in vivo, European cells \& materials 19 (2010) 30-40.

[54] A. Lode, C. Wolf-Brandstetter, A. Reinstorf, A. Bernhardt, U. Konig, W. Pompe, M. Gelinsky, Calcium phosphate bone cements, functionalized with VEGF: release kinetics and biological activity, J. Biomed. Mater. Res. Part A 81A(2) (2007) 474-483.

[55] E.K. Moioli, P.A. Clark, M. Chen, J.E. Dennis, H.P. Erickson, S.L. Gerson, J.J. Mao, Synergistic Actions of Hematopoietic and Mesenchymal Stem/Progenitor Cells in Vascularizing Bioengineered Tissues, PLoS One 3(12) (2008).

[56] K. Verma, J. Gu, E. Werner, Tumor Endothelial Marker 8 Amplifies Canonical Wnt Signaling in Blood Vessels, PLoS One 6(8) (2011) e22334.

[57] J. Wilting, B. Christ, M. Bokeloh, H. Weich, In vivo effects of vascular endothelial growth factor on the chicken chorioallantoic membrane, Cell and Tissue Research 274(1) (1993) 163-172.

[58] J. Wilting, B. Christ, H.A. Weich, The effects of growth factors on the day 13 chorioallantoic membrane (CAM): a study of VEGF165 and PDGF-BB, Anatomy and embryology 186(3) (1992) 251-7.

[59] D. Lindhorst, F. Tavassol, C. von See, P. Schumann, M.W. Laschke, Y. Harder, K.H. Bormann, H. Essig, H. Kokemuller, A. Kampmann, A. Voss, R. Mulhaupt, M.D. Menger, N.C. Gellrich, M. Rucker, Effects of VEGF loading on scaffold-confined vascularization, J. Biomed. Mater. Res. Part A 95A(3) (2010) 783-792.

[60] A. Alfranca, VEGF therapy: a timely retreat, Cardiovascular Research 83(4) (2009) 611-612.

[61] L. Urbani, C. Camilli, D.E. Phylactopoulos, C. Crowley, D. Natarajan, F. Scottoni, P. Maghsoudlou, C.J. McCann, A.F. Pellegata, A. Urciuolo, K. Deguchi, S. Khalaf, S.F. Aruta, M.C. Signorelli, D. Kiely, E. Hannon, M. Trevisan, R.R. Wong, M.O. Baradez, D. Moulding, A. Virasami, A. Gjinovci, S. Loukogeorgakis, S. Mantero, N. Thapar, N. Sebire, S. Eaton, M. Lowdell, G. Cossu, P. Bonfanti, P. De 
Coppi, Multi-stage bioengineering of a layered oesophagus with in vitro expanded muscle and epithelial adult progenitors, Nat Commun 9(1) (2018) 4286. 


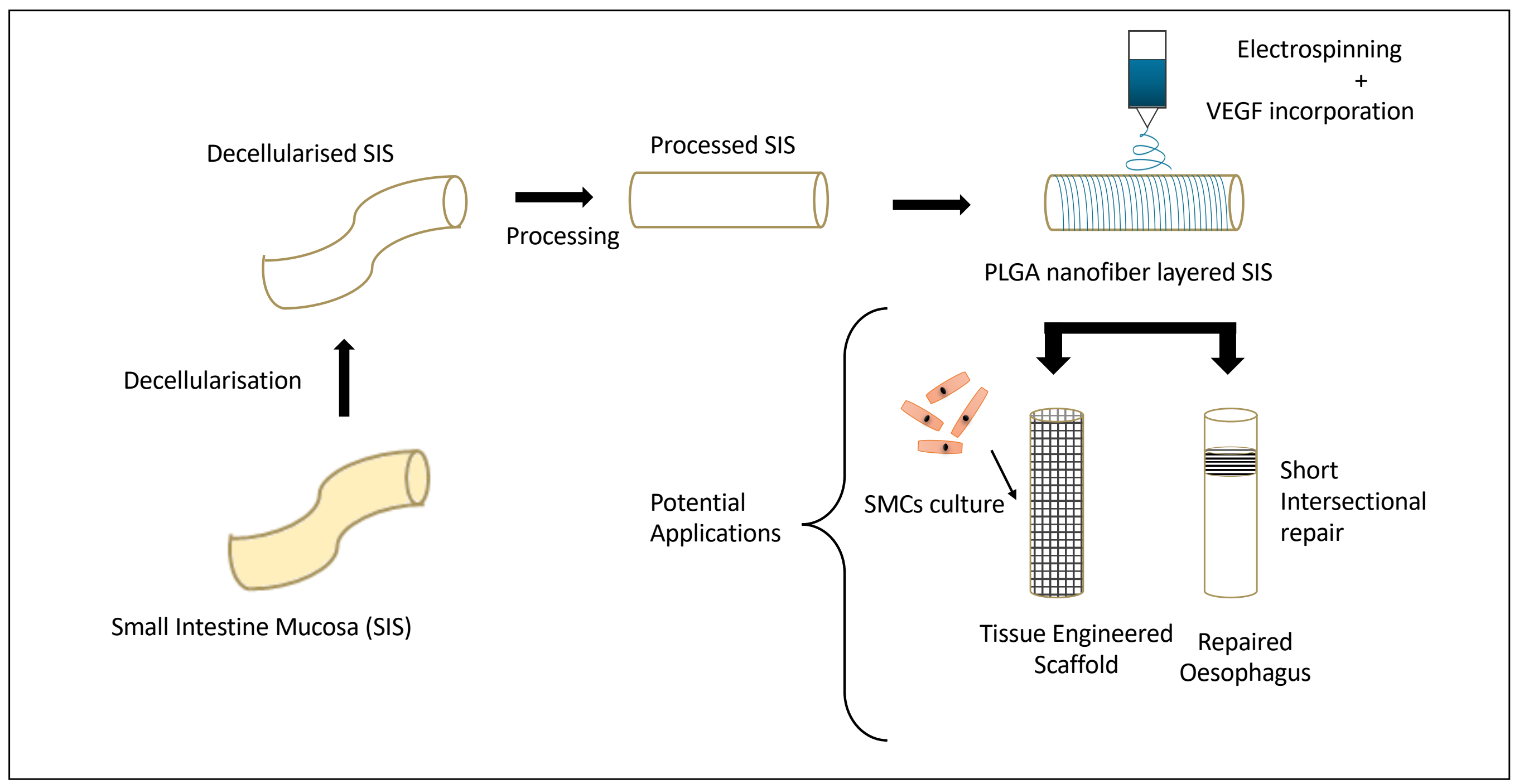

Figure 1 Schematic illustrating the various stages of the development of hybrid PLGA-SIS scaffolds and potential applications for oesophageal tissue engineering 
$10 \mathrm{rpm}$
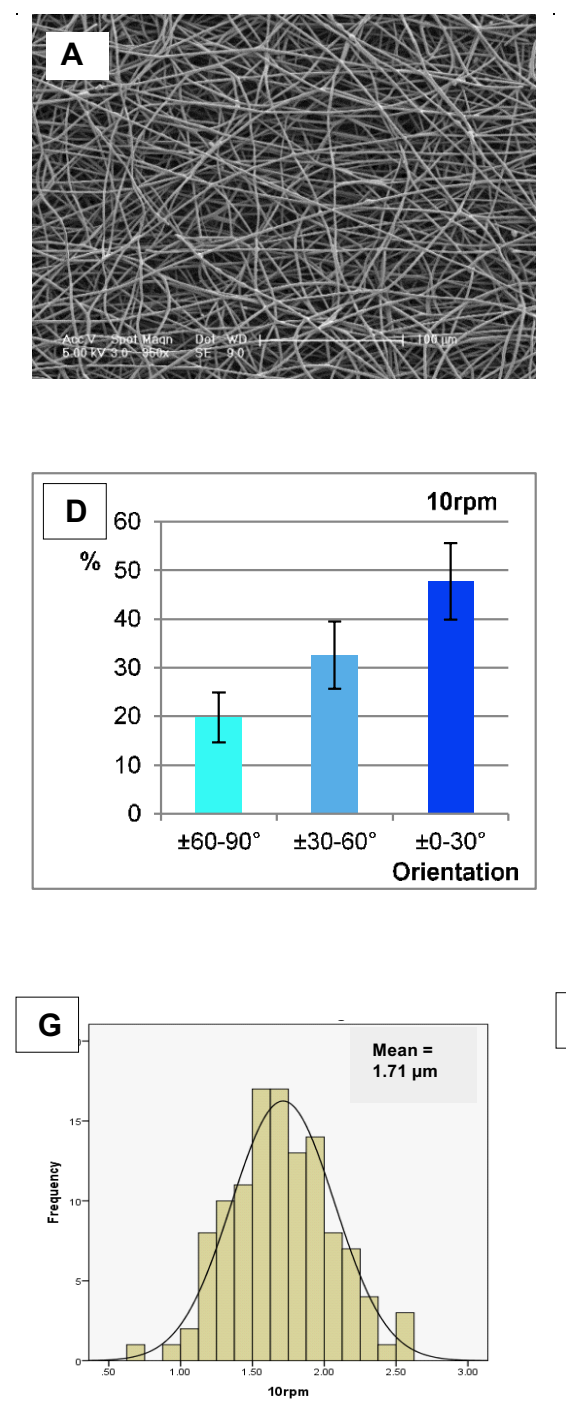

$3000 \mathrm{rpm}$
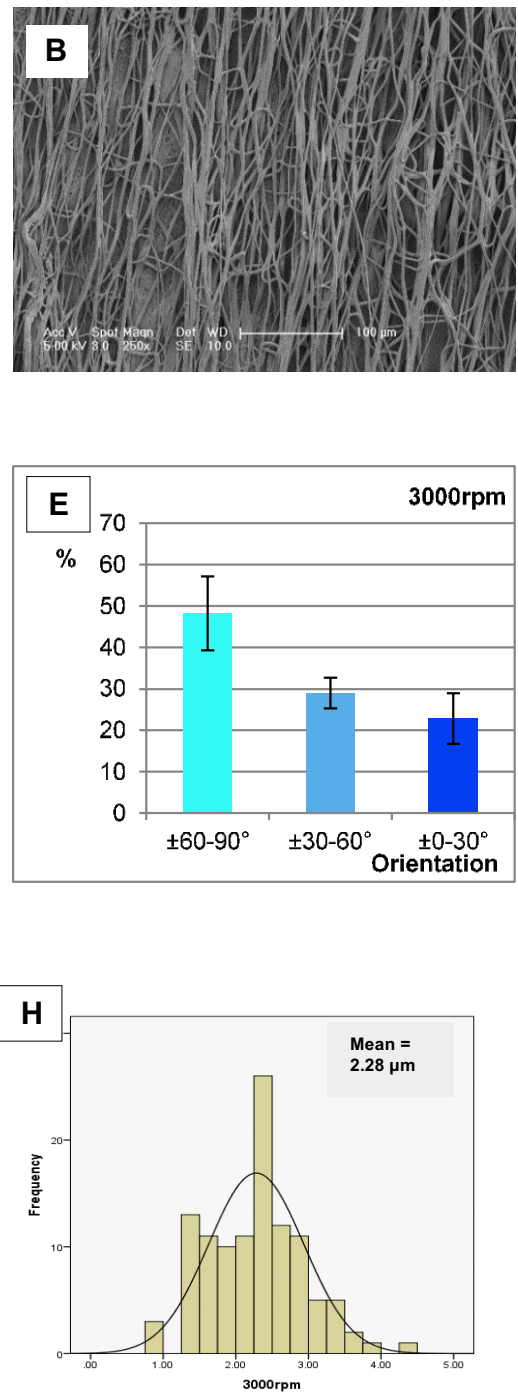

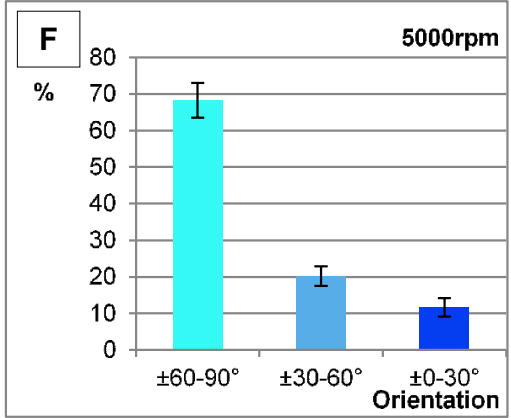

$5000 \mathrm{rpm}$
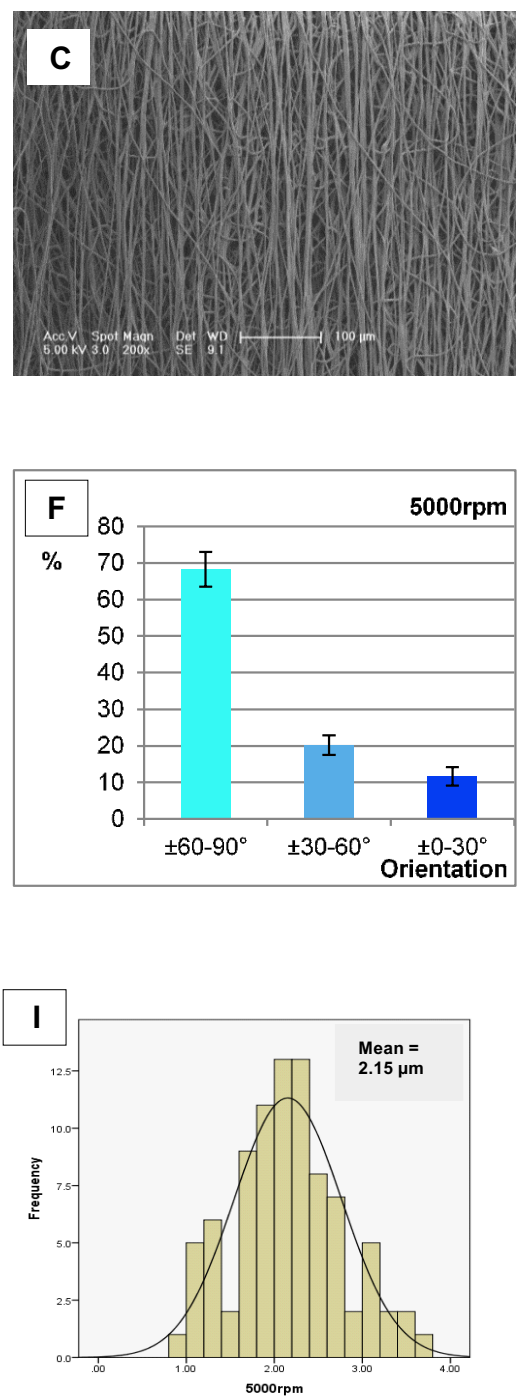

Figure 2 SEM images (A)-(C) PLGA fibres at mandrel rotational speeds of 10,3000 \& $5000 \mathrm{rpm}$ (D)-(F) PLGA fibre orientation at 10, 3000,5000 rpm in terms of the percentage of fibres in each of 3 groups spanning $0-90^{\circ}$ from the mandrel axis of rotation $(n=10-20)$. The error bars are standard deviation. (G) - (I) Frequency histograms for the fibre diameter ( $\mathrm{um}$ ) of the PLGA fibres produced at $10,3000,5000 \mathrm{rpm}$. The histograms are overlaid with an ideal normal distribution and the mean values $(n=50+)$. 

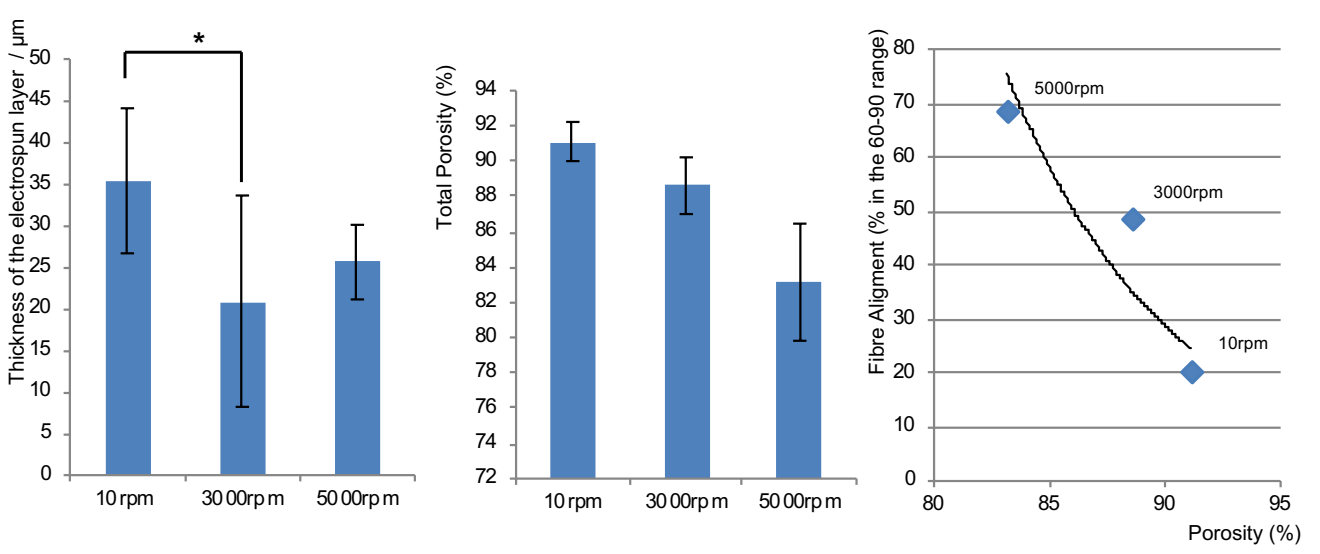

Figure 3 (a) The average thickness of the PLGA electrospun layers for the three alignment groups $(n=15+)$. The error bars are standard deviation. Important values differing significantly from each other are denoted by ${ }^{*} ; p<0.05$. (b) Graph are standard deviation. (c) Graph illustrating the potential trend between fibre alignment and the porosity of the hybrid scaffold groups. 


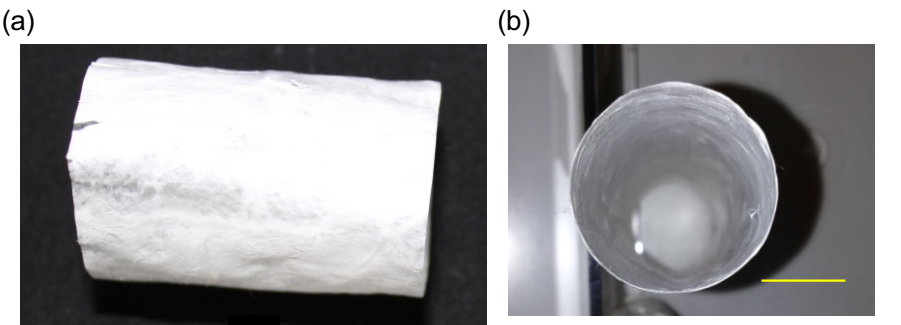

I

(c)

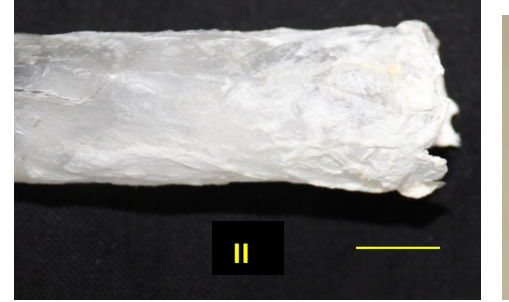

Figure 4 Images showing (a) the two different sizes of tubing used for processing. I - Working method (2.54 cm diameter) and II - Earlier method (1.9

$\mathrm{cm}$ diameter). The change in diameter resulted in improving the surface

smoothness of the SIS due to the stretching effect by the larger tube. (b) The

smooth and regular shape of the final SIS scaffold (c) final hybrid scaffold (5000 rpm sample) scale bars are $1 \mathrm{~cm}$. 


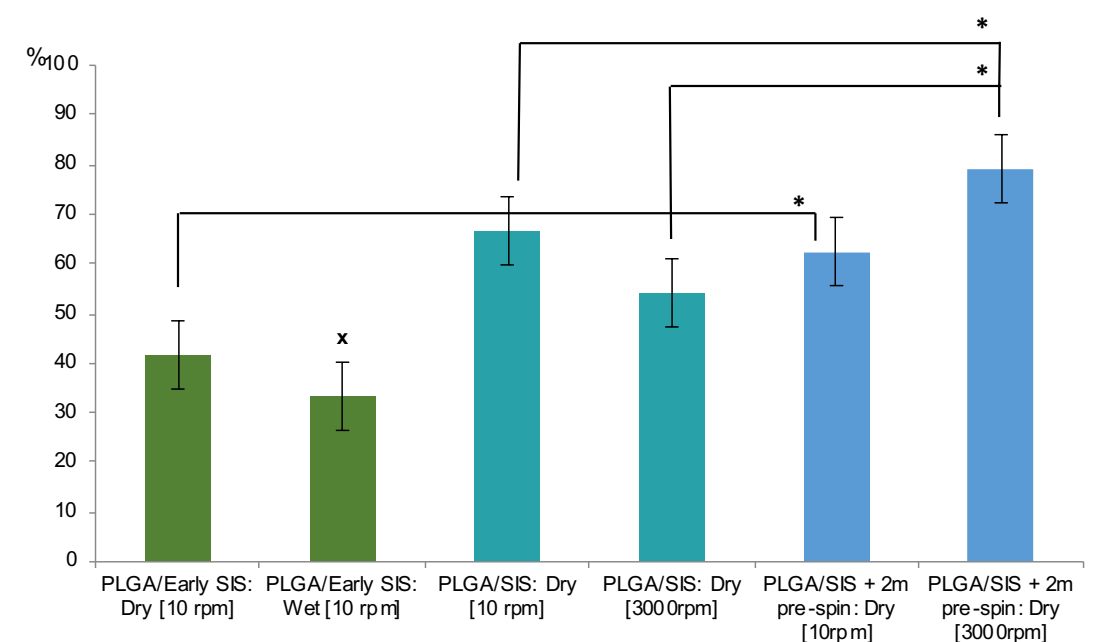

Figure 5 The percentage of samples where the SIS and the electrospun layers remained attached. Groups shown include "PLGA/Early SIS: Dry" and "PLGA/Early SIS. Wet" $(n=24)$ using the earlier method with the smaller diameter. The remaining 4 groups all used the standard sis used in all other work ( $=24$ ). Imporant values ding 4 groups all used the to the practical difficulties in achieving attachment 


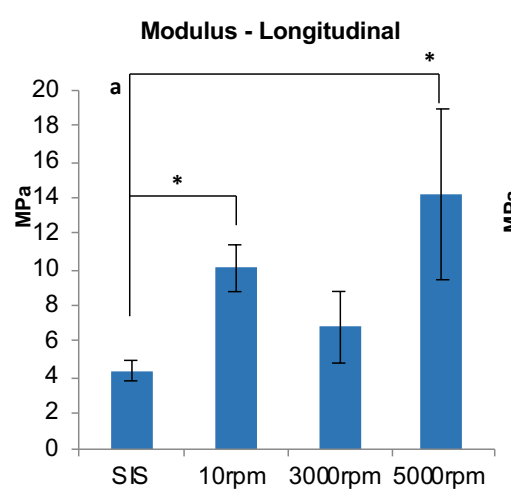

Yield Stress - Longitudinal

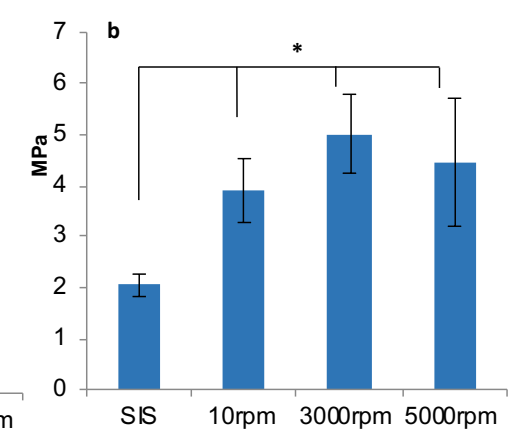

Yield Stress - Circumfrential

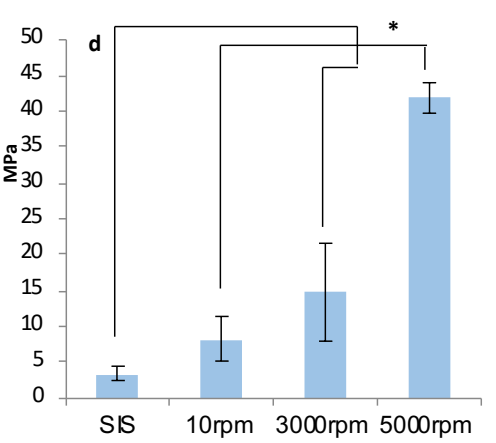

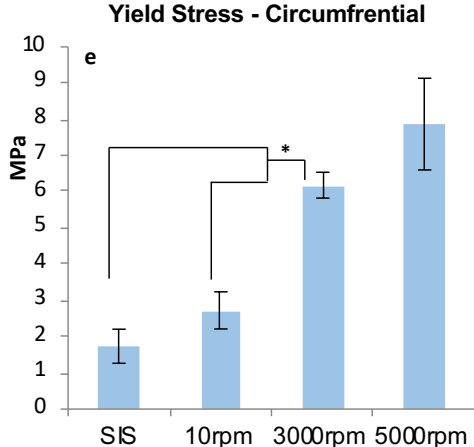

SIS $10 \mathrm{rpm} 3000 \mathrm{rpm} 5000 \mathrm{rpm}$
Failure Strain - Longitudinal
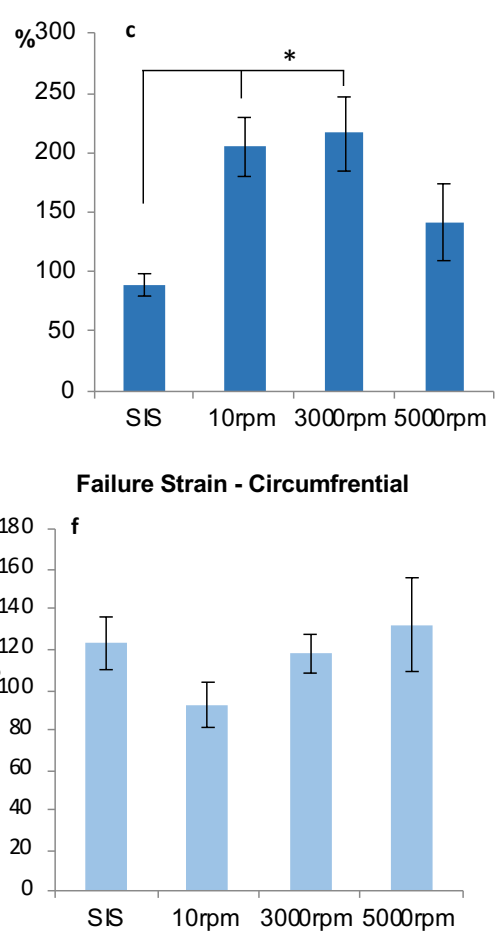

Figure 6 (a) - (c) Mechanical testing data from the longitudinal tensile testing of the hybrid scaffold groups: (a) Elastic modulus values (MPa) $(n=12)$ (b) Yield stress values (MPa) $(n=12)$ (c) Failure strain values $(\%)(n=12)$. (d) - (f) Mechanical testing data from the circumferential tensile testing of the hybrid scaffold groups: (d) Elastic modulus values (MPa) $(n=8)$ (e) Yield stres

n-parametrically and discussed in the results sections. 
(a)

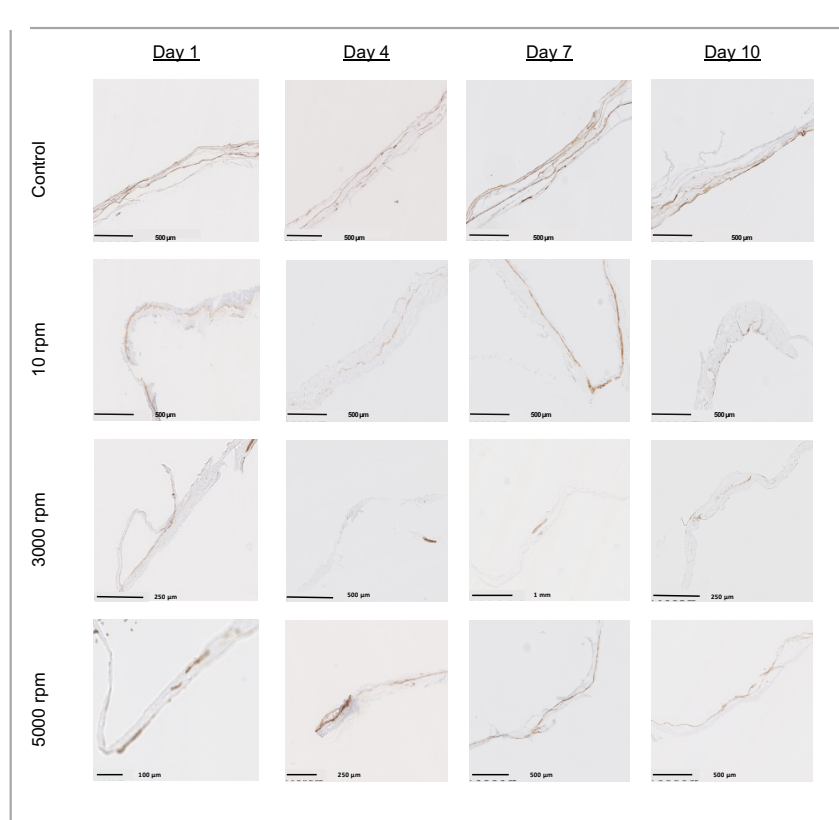

(b)

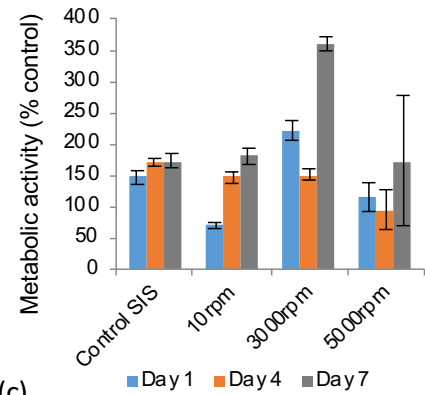

(c)

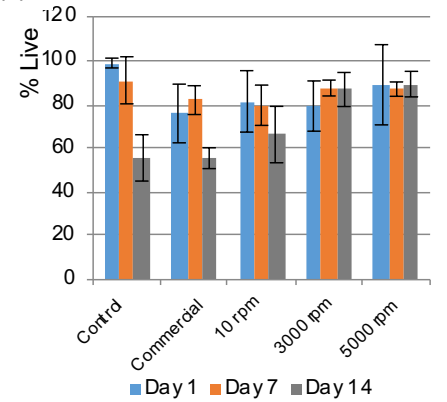

(d)

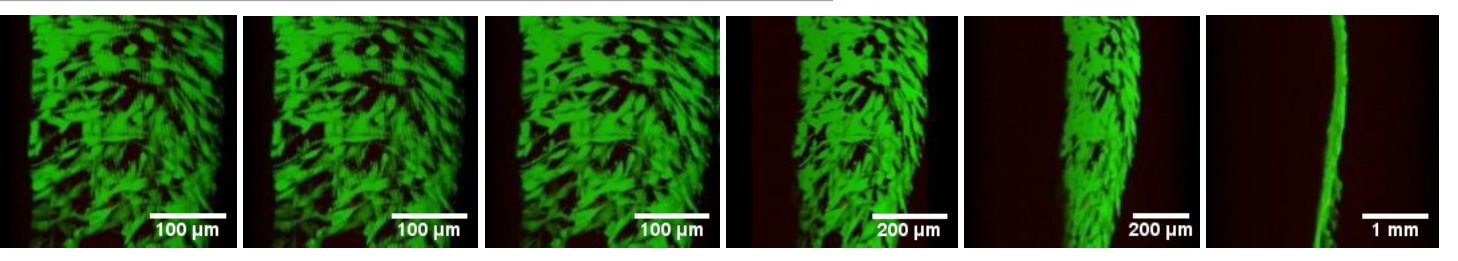

Figure 7(a) Histological images demonstrating positive IHC staining for smooth muscle actin (SMA) from HESMC's cultured on the commercial control SIS in addition to the hybrid scaffold groups (10,3000 and $5000 \mathrm{rpm})$ at Days $1,4,7$ and 10. (b) Graph representing the normalised levels of metabolic activity of HESMC's cultured on the hybrid scaffold groups. The values are expressed as a percentage of the control (in this case the HESMC's cultured on TCP). (c) Graph representing the percentage of live cells observed by performing a Live-Dead assay on HESMC's cultured on the scaffold groups. The values were obtained cell counts from confocal microscopy images and image stacks ( $n$ is between 9 and 20 images or image stacks). (d) Series of confocal images (10 rpm) showing the 3D projections of a stack of confocal images for the purpose of live-dead analysis at Day 7 


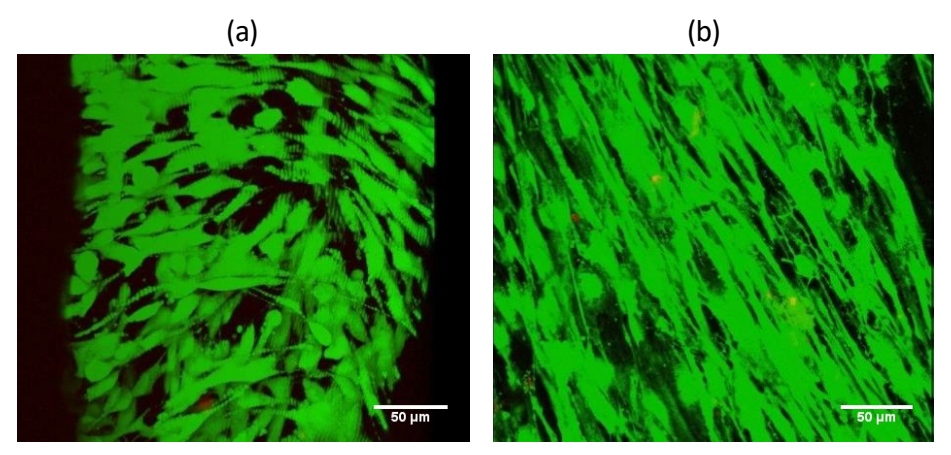

Figure 8 Confocal images (Calcein AM and Propidium lodide (PI)) demonstrating different cell orientations: (a) random - $10 \mathrm{rpm}$ at day 7 and (b) aligned cells -500 $\mathrm{rpm}$ at day 14. Scale bars are $50 \mu \mathrm{m}$ 


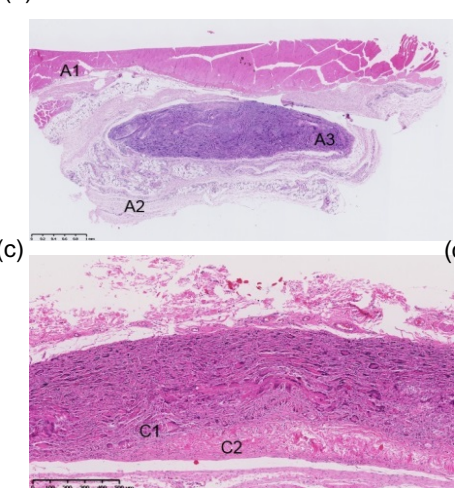

(f)
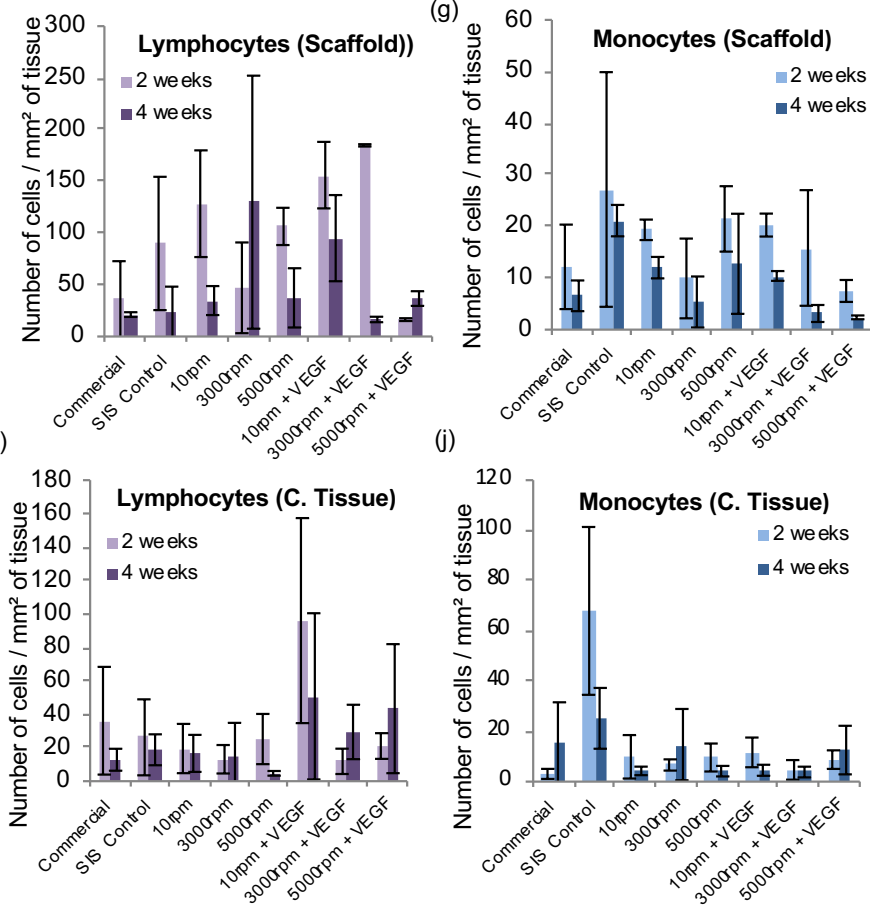

(k)

200 FBGC (Scaffold)

为 180

¿ 140 - $=4$ weeks

है 120

응 80

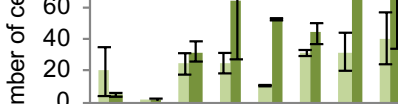

$\sum$ z
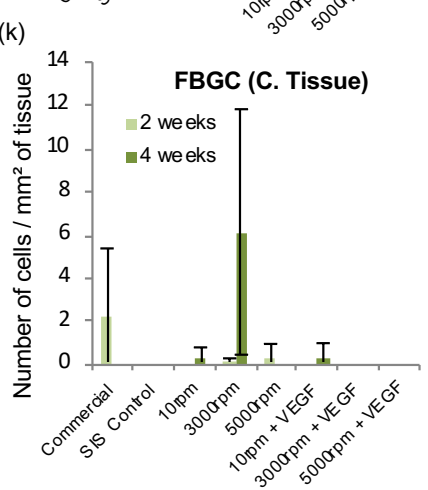

Figure 9 Histological images (H\&E) illustrating general observations of the in vivo samples (a) Typical features (3000rpm - 4 weeks) demonstrating the (A1) underlying muscular tissue (A2) surrounding granulation tissue (A3) encapsulated scaffold (b) Pure SIS sample (Day 7), B1-Pink stained collagen-based SIS which is well infiltrated, B2- Boundary between the capsule and the underlying muscle tissue

(c) PLGA-SIS 5000 rpm (Day7), C1-Well infiltrated PLGA Fibres, C2- Well infiltrated SIS (c) PLGA-SIS 5000 rpm (Day7),

(d) D1-Poorly infiltrated electrospun fibres, and D2- Poorly infiltrated SIS

(e) Graph of the mean cellular infiltration score. The images were scored from 0-4 with 0 being poor infiltration and 4 being highly infiltrated ( $n=4$ and the error bars are standard deviation).

Graphs $(g-h)$ : The average number of cells per $\mathrm{mm} 2$ of histologically visible scaffold for the following the cell types: (f) Lymphocytes (g) Monocytes ( $h$ ) FBGC's ( $n=3$ and the error bars are the standard deviation).

Graphs (i-k): The average number of cells per $\mathrm{mm} 2$ of histologically visible sounding connective tissue for the following the cell type (d) Lymphocytes (e) Monocytes (f) FBGC's ( $n=3$ and the error bars are the standard deviation). 

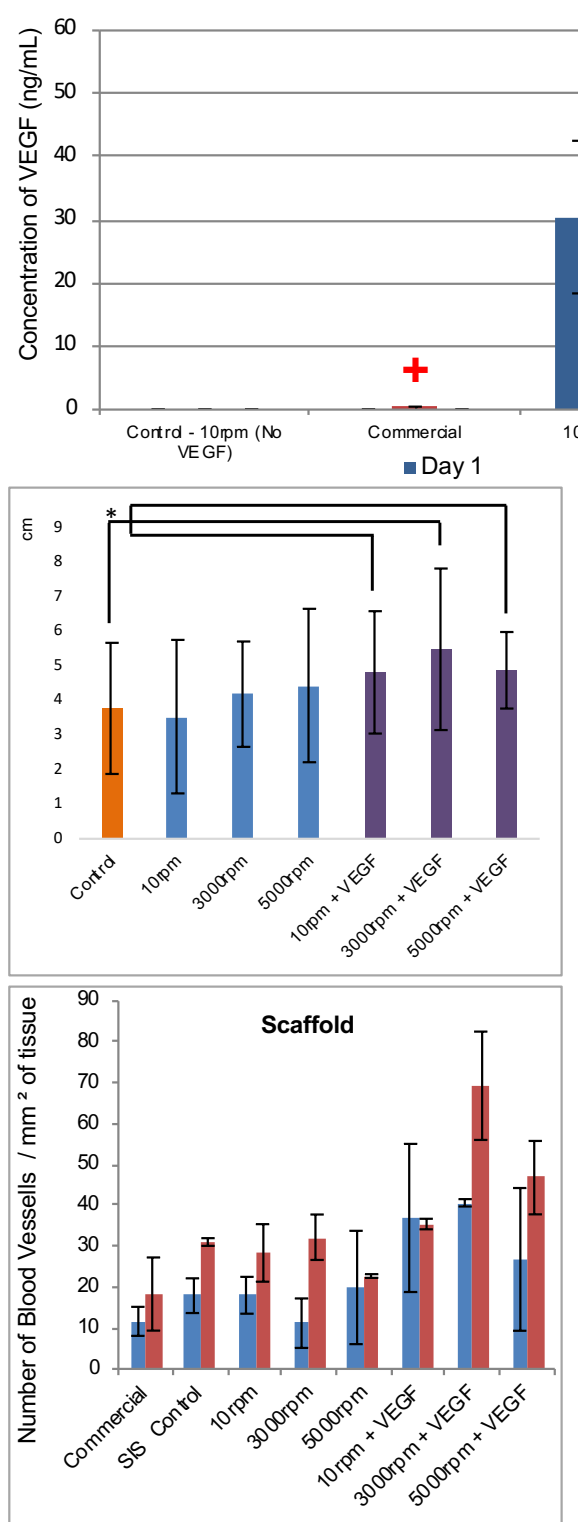

2 Weeks -4 weeks

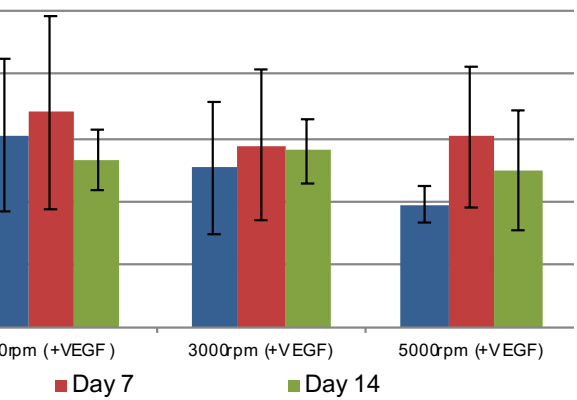

- Day 7

Day 14
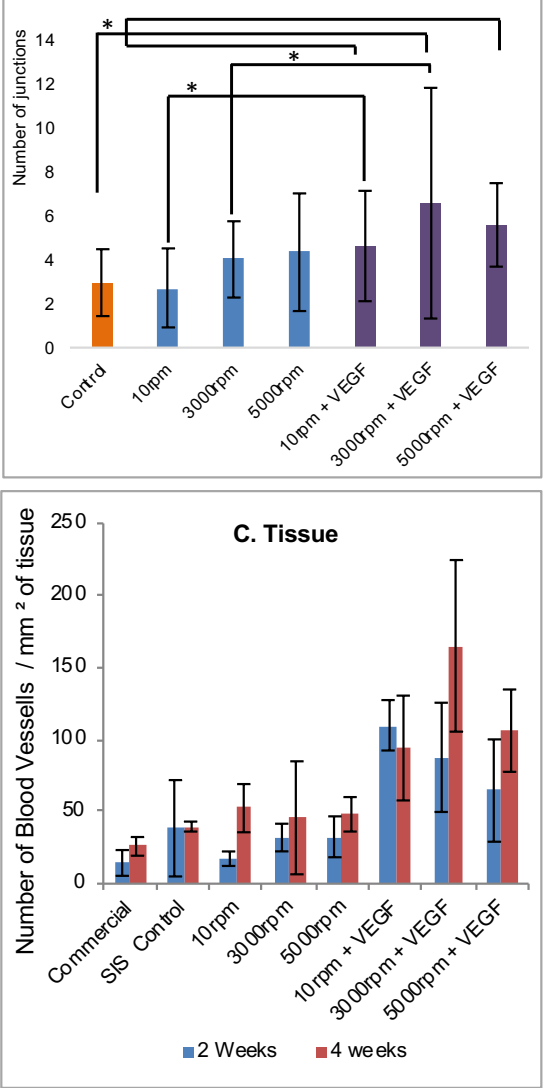

Figure 10 (a) Graph representing the concentration of VEGF in solution following hybrid scaffold immersion at day intervals of 1, 7 and 14, along with a commercial SIS and a control (in this instance $10 \mathrm{rpm}$ with no VEGF). The "+" denotes a trace amount of VEGF detected in the commercial VEGF on day $7(n=4$ and the error bars are standard deviation). (b) The mean length of vessel complex on the CAM for the different scaffold groups as identified by quantification software. The sample groups shown are (with $n$ number in brackets) : Control (24), $10 \mathrm{rpm}$ (21), $3000 \mathrm{rpm}$ (15), $5000 \mathrm{rpm}$ (21), $10 \mathrm{rpm}+$ VEGF (20), $3000 \mathrm{rpm}+$ VEGF (26), and $5000 \mathrm{rpm}+$ VEGF (25). (c) The mean number junctions found per vessel complex for the different scaffold groups on the CAM as identified by quantification software. The sample groups shown are: Control (24), $10 \mathrm{rpm}$ (21), $3000 \mathrm{rpm}$ (15), $5000 \mathrm{rpm}$ (21), 10 $\mathrm{rpm}+$ VEGF (20), $3000 \mathrm{rpm}+\mathrm{VEGF}(26)$, and $5000 \mathrm{rpm}+$ VEGF (25). All error bars are the standard deviation. Important values differing significantly from each other are denoted by $*$; $<<0.05$. The average number of histologically visible blood vessels per $\mathrm{mm}^{2}$ of tissue based on histological analysis $(n=4$ for the number of sample images and the error bars are the standard deviation). The bars are alternatively shaded between groups at weeks 2 and 4 . 
(a)

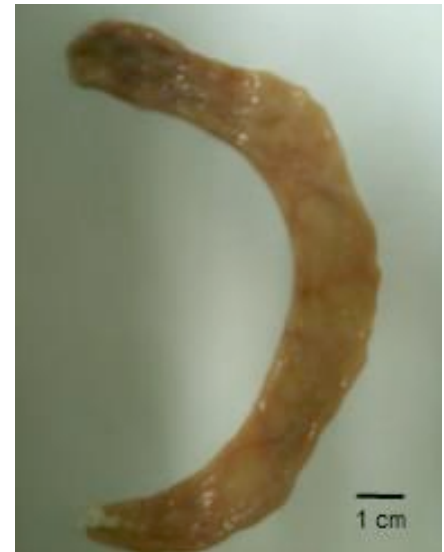

(b)

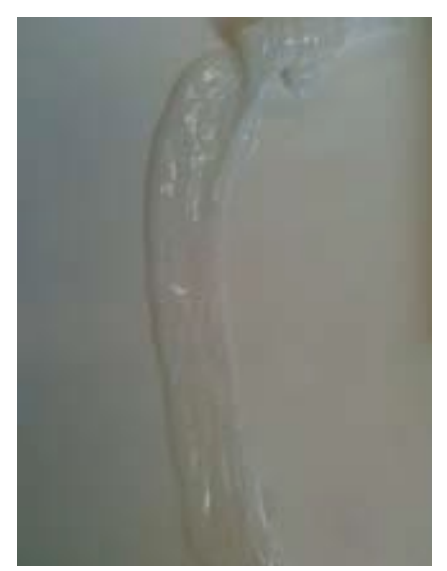

(c)

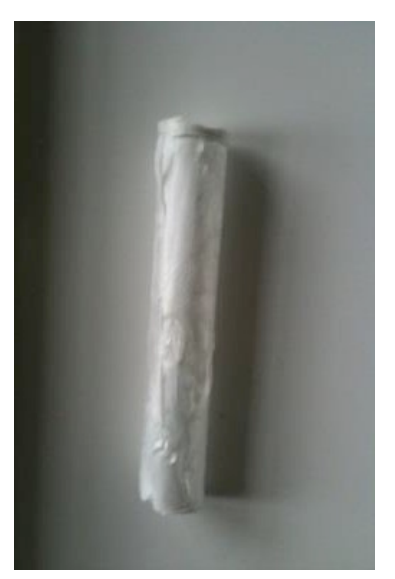

(d)

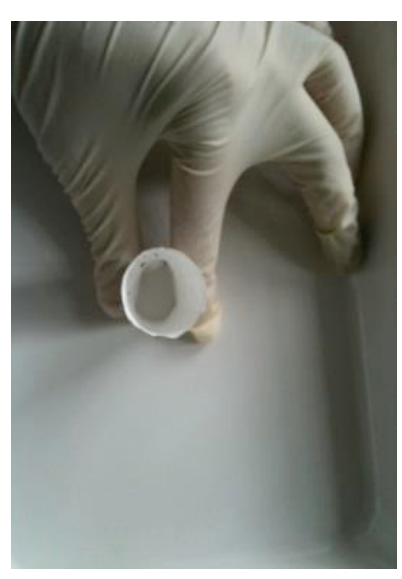

(e)

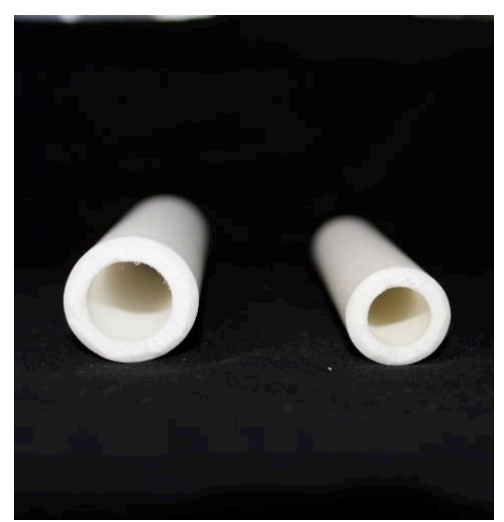

Supplementary 1: Stages of SIS preparation (a) Porcine small intestine section prior to debridement (b) SIS following decellularisation (c) \& (d) Final processed form of SIS (e) Image of porous polyethylene solid tubes highlighting the size difference the cross-section. $2.54 \mathrm{~cm}$ diameter tube and $1.9 \mathrm{~cm}$ diameter tube 

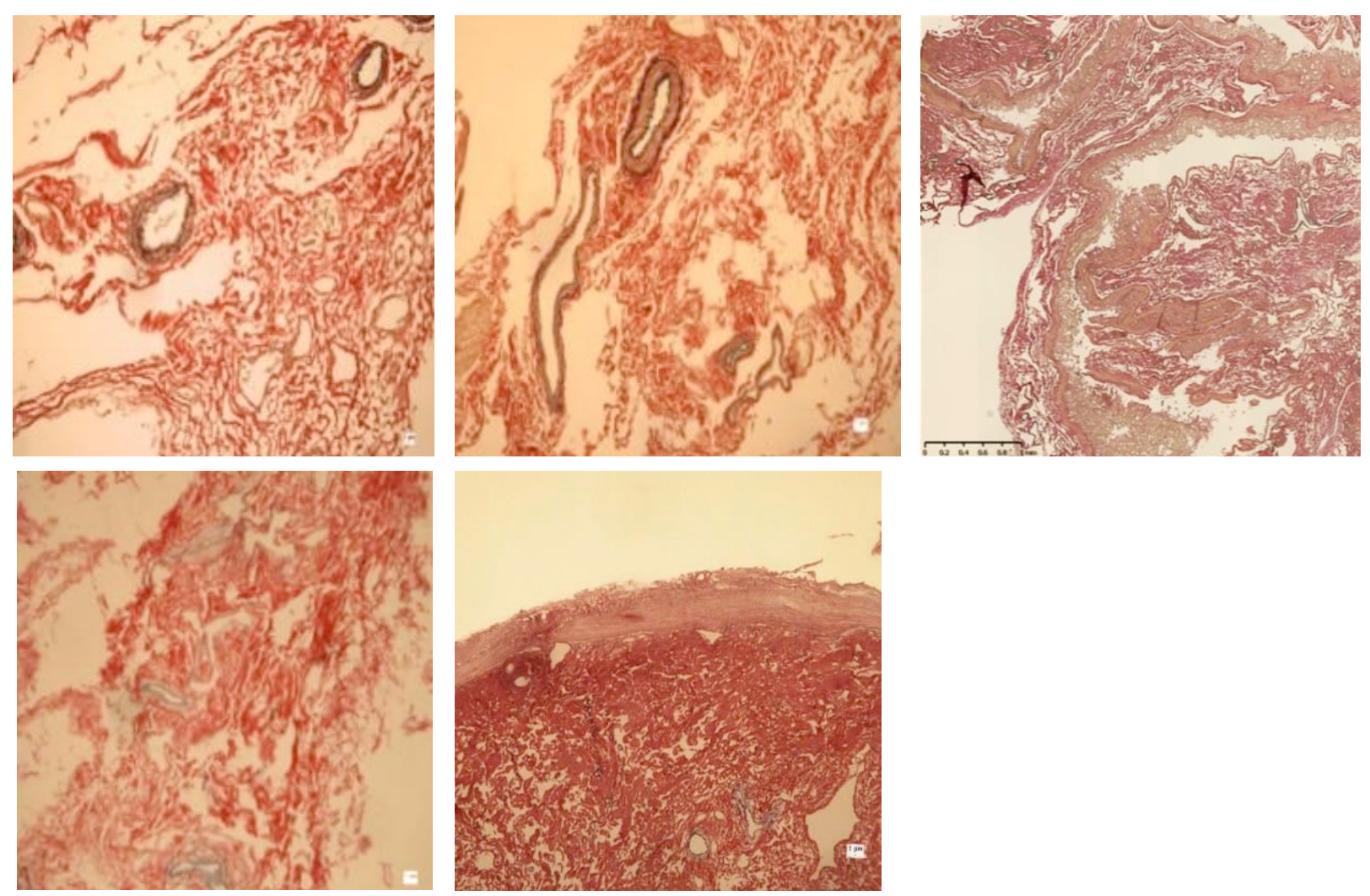

Supplementary 2 Histological images of decellularising SIS and the controls (stained with EVG) (a) uSIS (b) PAA-2hA (c) PAA-12h (d) SD/DN (e) SDS/TX 

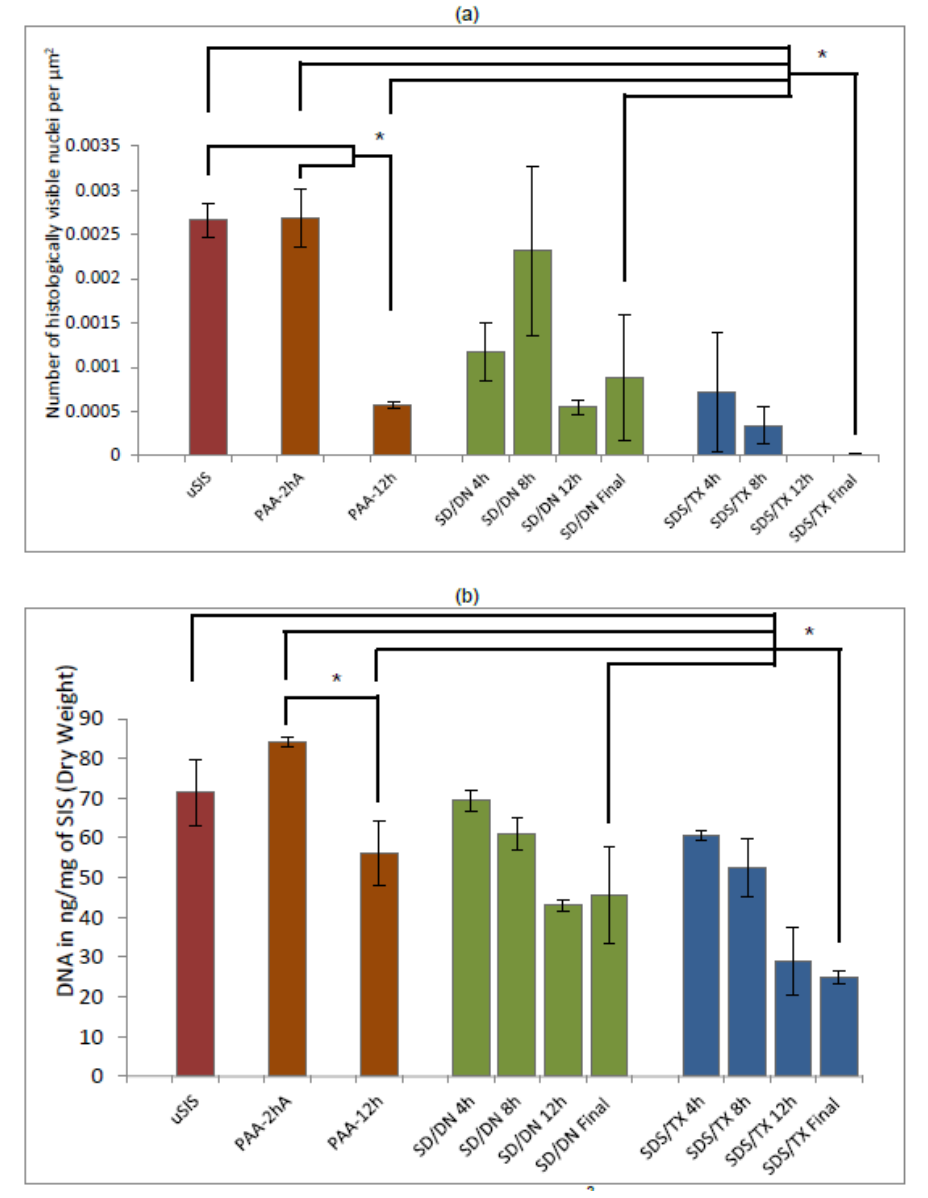

Supplementary 3 (a) The average number of distinct nuclei counted per $\mu \mathrm{m} 2$ of SIS for all groups. Analysis was carried out on images taken of H\&E stained histological sections, with random sites chosen and the nuclei counted using software tools (Image $\mathrm{J})(n=10-20)$. All counts were then averaged together for each stage. Error bars are standard error. (b) DNA quantification values ( $\mathrm{ng} / \mathrm{mg}$ of dry weight) for the all three protocols and the control with each column representing the concentration of DNA at various time-points in the decellularising protocols including the processed SIS $(n=4)$. Error bars are the standard error. Important values differing significantly from each other are denoted by *; $p<0.05$. 


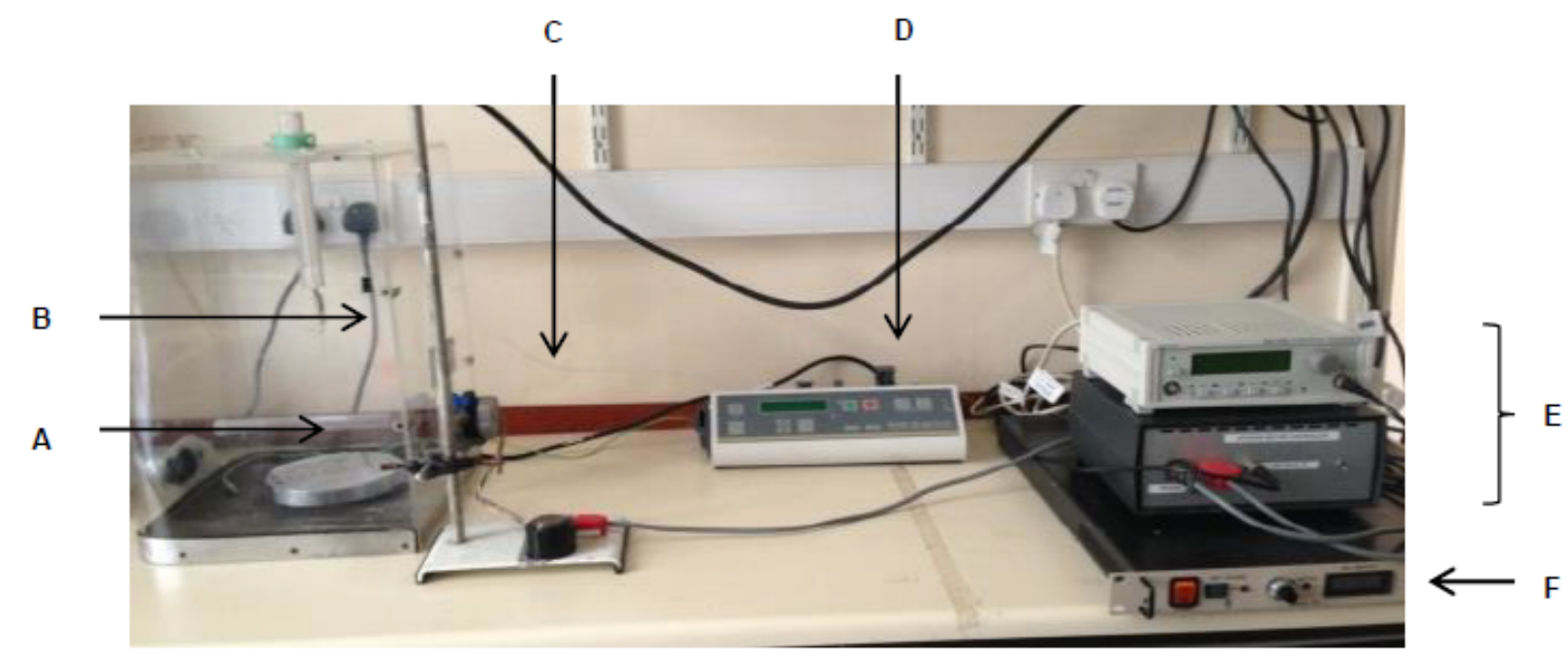

Supplementary 4: Electrospinning arrangement used in Series 1. A) Mandrel B)

Needle and power connector C) Step motor D) Syringe Pump E) Motor controller and switch F) Power Source 
(a)

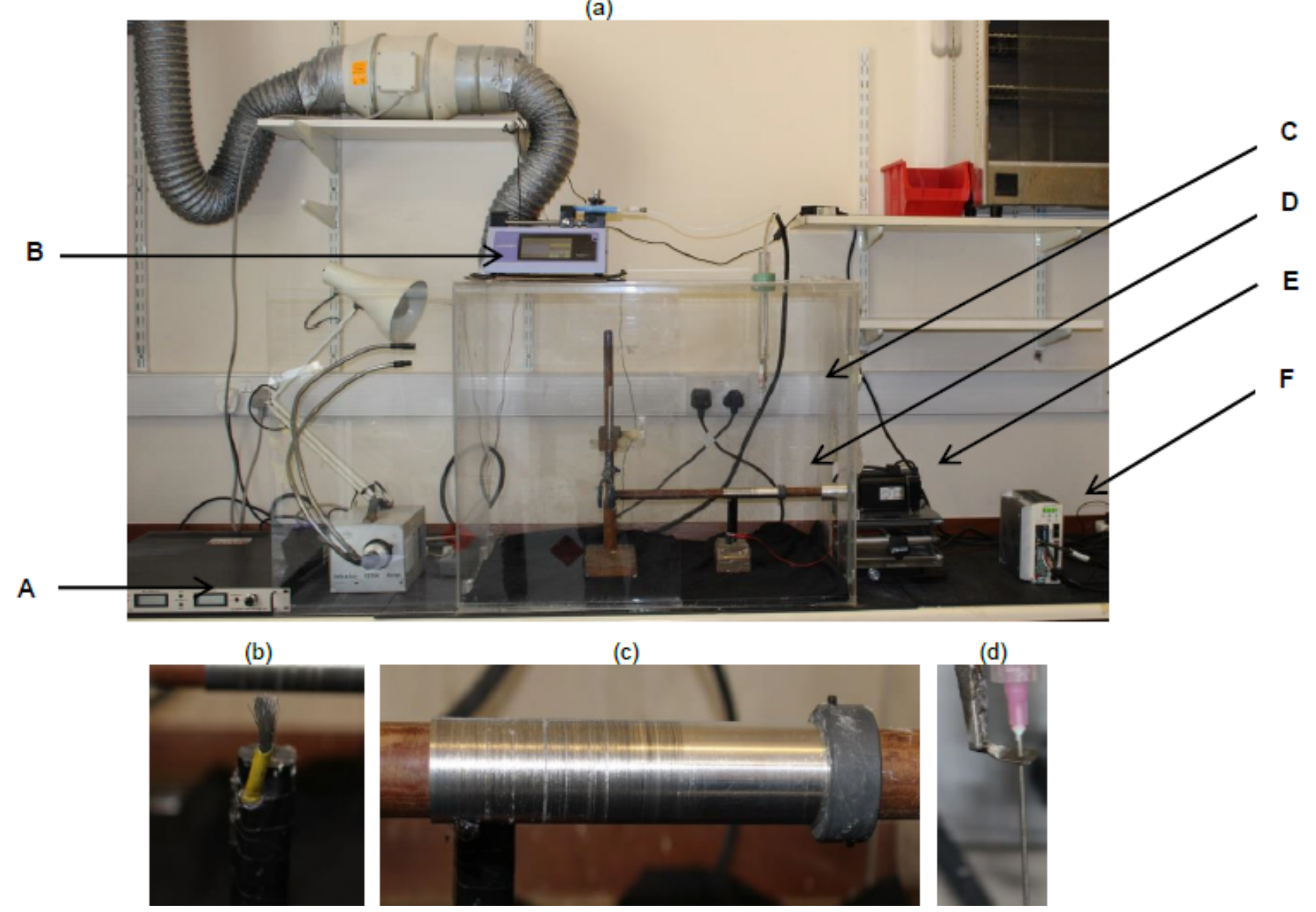

Supplementary 5 (a) Electrospinning arrangement used in Series 2: A) Power Source, B) Syringe Pump, C) Needle and power connector, D) Mandrel, E) Servo motor, F)Motor controller. (b) Brush ground contact (c) Custom collector (d) Needle and power arrangement 
(a)

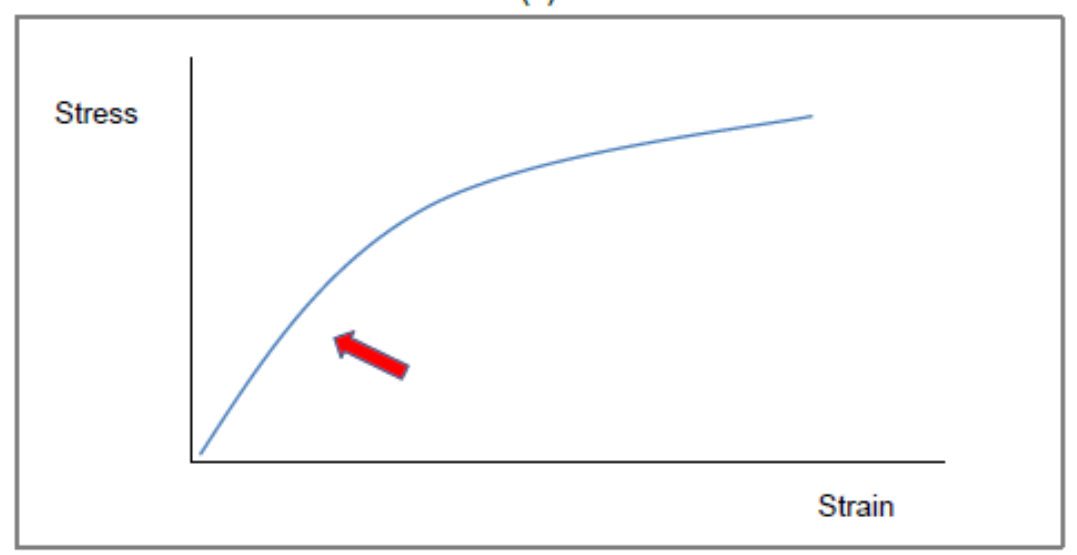

(b)

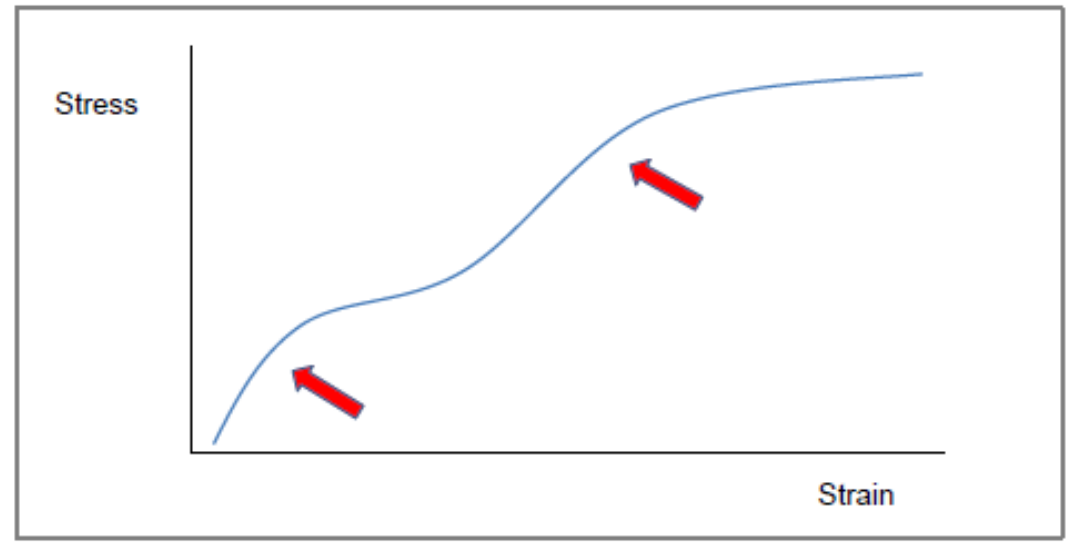

Supplementary 6 Generalised illustration of a typical stress-strain graph for (a) SIS and (b) PLGA-SIS hybrid materials. The red arrows denote the yield point occurring at the end of an elastic region of the stress-strain curve. 
(a)

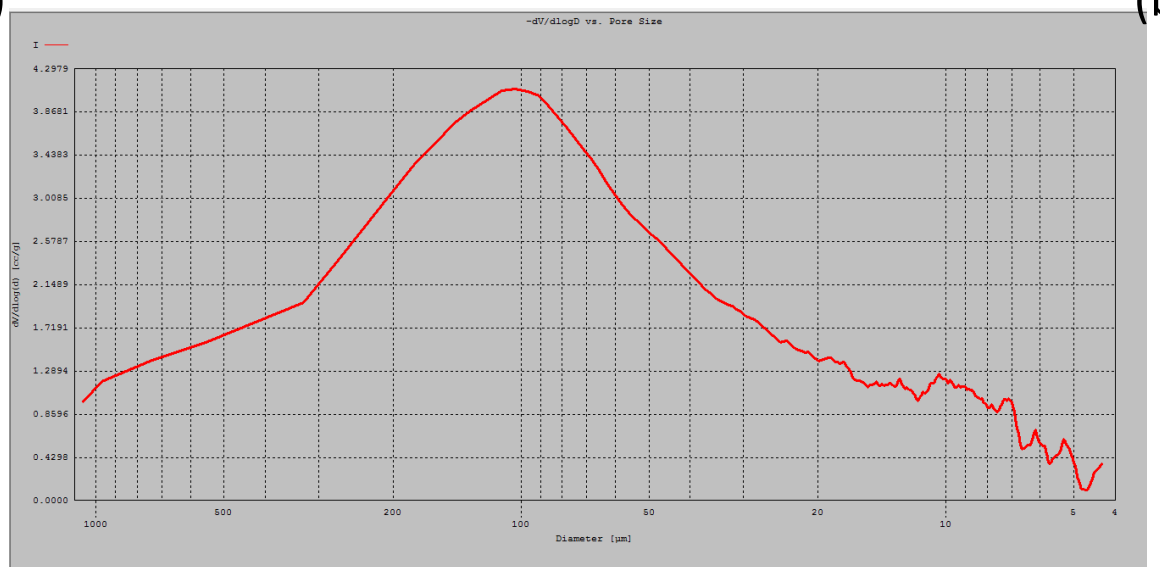

(b)

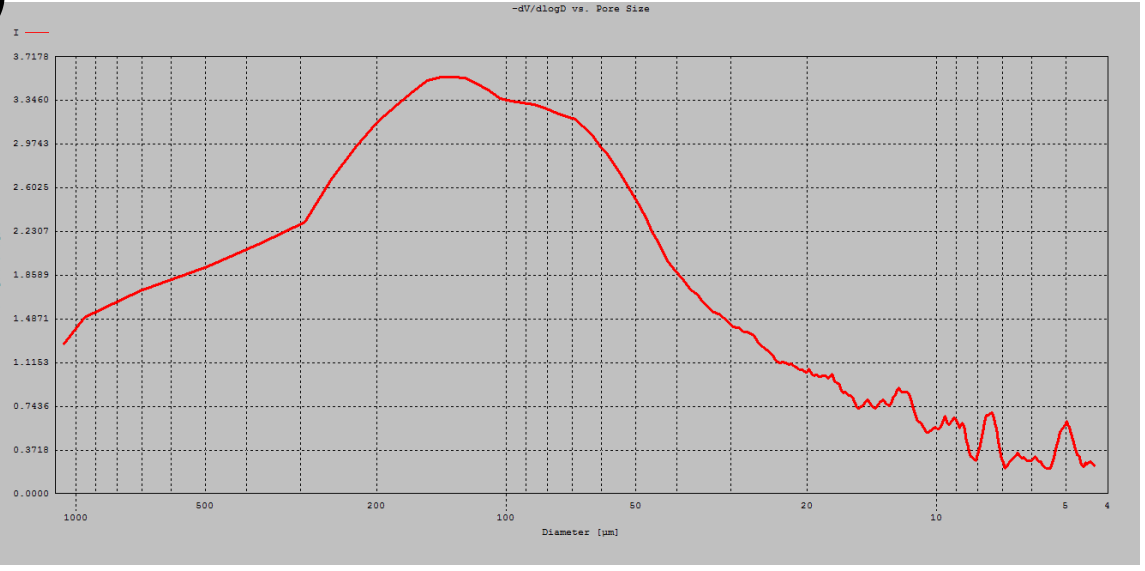

(c)

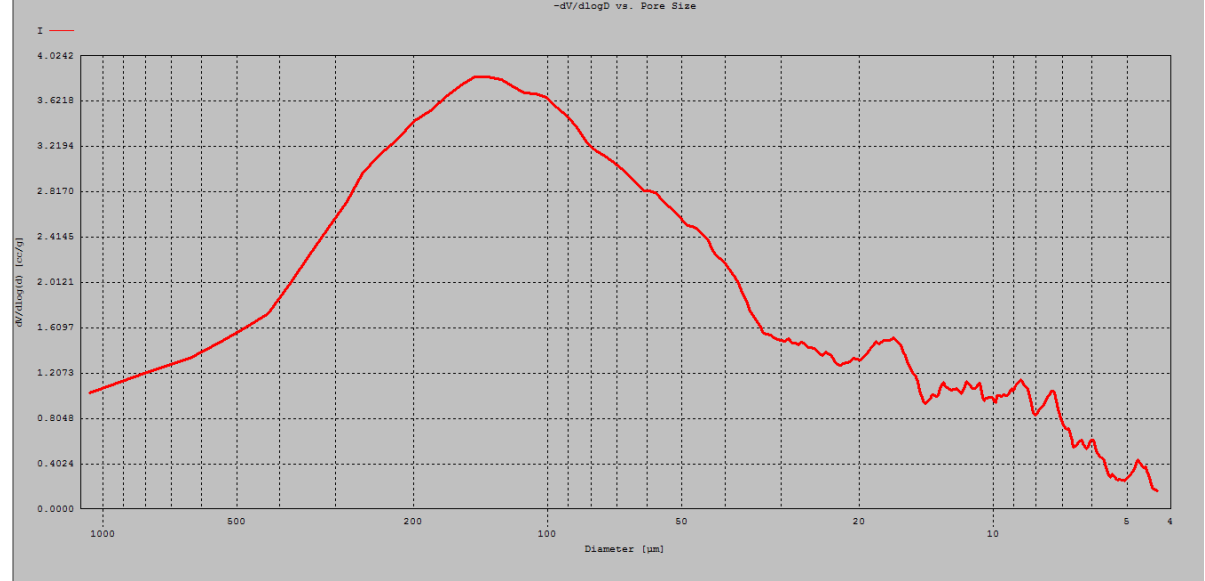

Supplementary 7 Pore size distribution for the scaffolds produced using different mandrel rotational speeds:

(a) b) (c) $10 \mathrm{rpm}$ The left of the $x$-axis represents the diameter of the pores in $\mu \mathrm{m}$ 
(d)

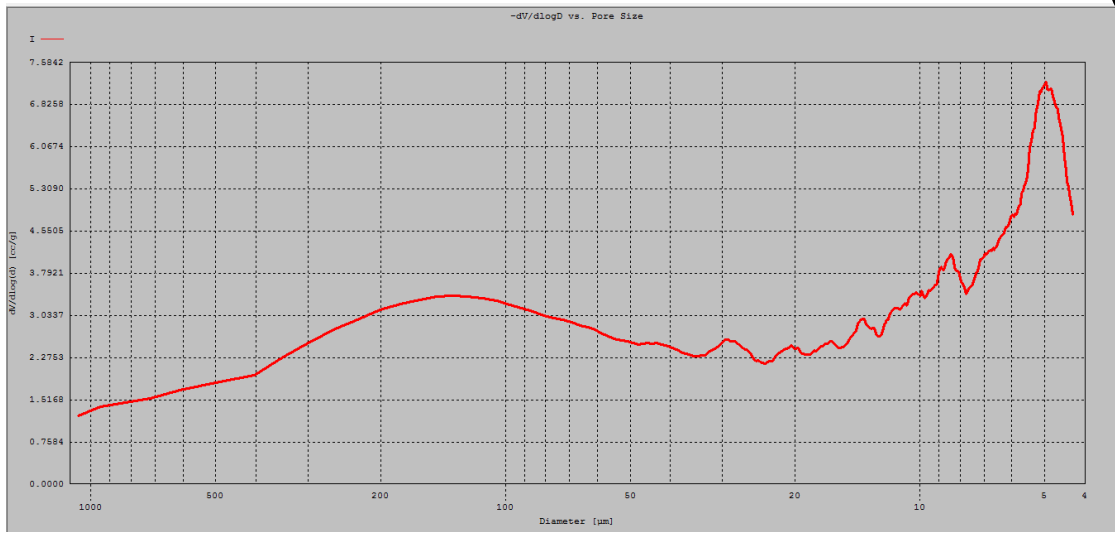

(e)

(f)

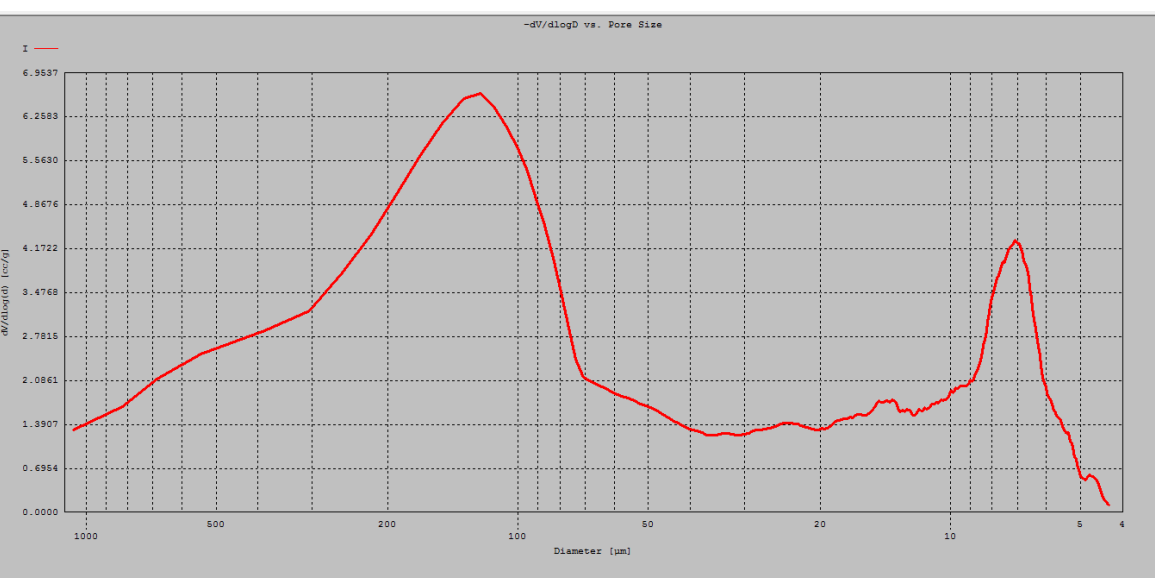

Supplementary 8 Pore size distribution for the scaffolds produced using different mandrel rotational speeds: (d) (e) (f) $3000 \mathrm{rpm}$ (g) (h) (i) $5000 \mathrm{rpm}$. The left of the x-axis

represents the diameter of the pores in $\mu \mathrm{m}$ 

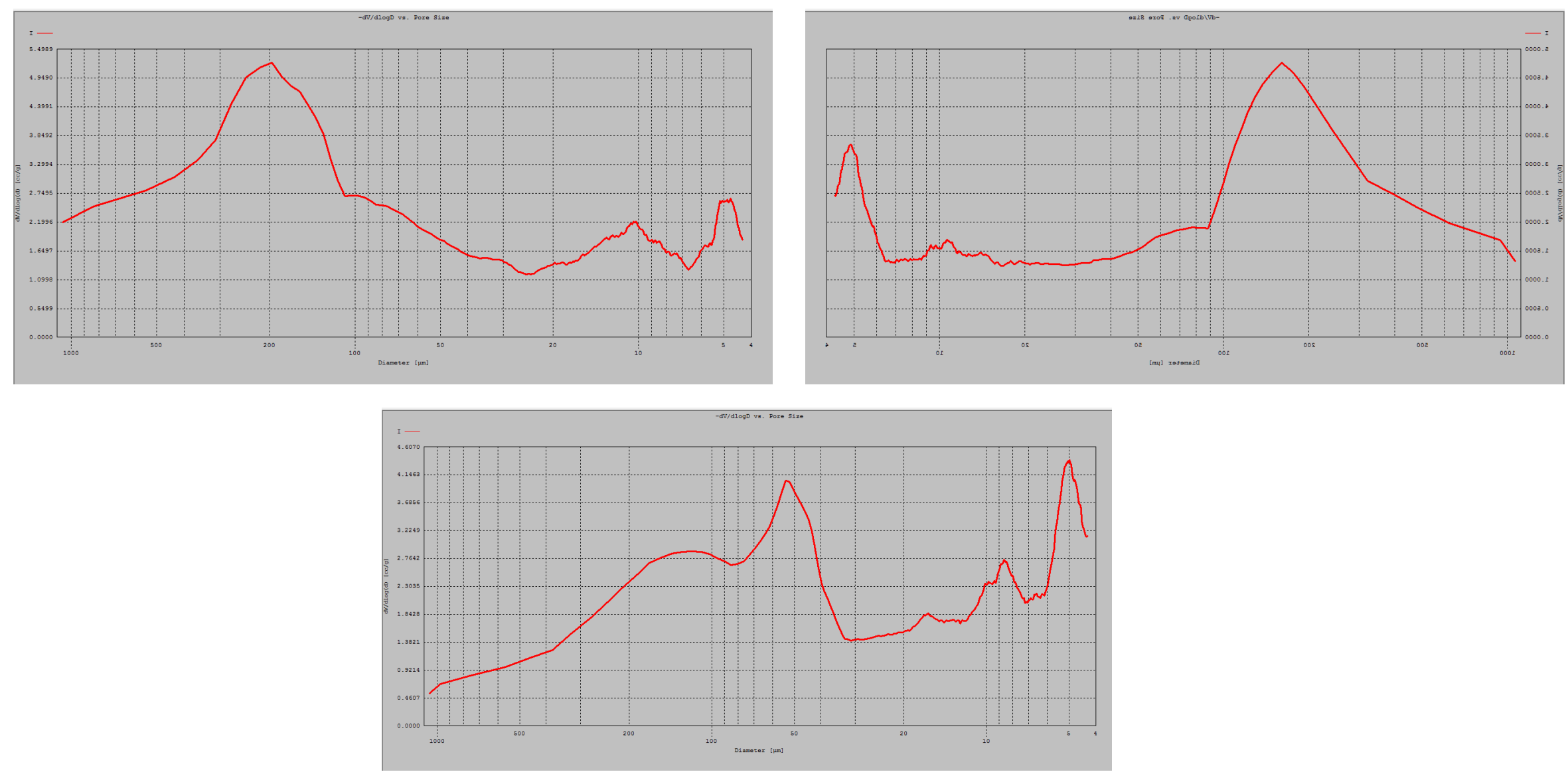

Supplementary 9 Pore size distribution for the scaffolds produced using different mandrel rotational speeds: (g) (h) (i) $5000 \mathrm{rpm}$. The left of the $x$-axis represents the diameter of the pores in $\mu \mathrm{m}$ 

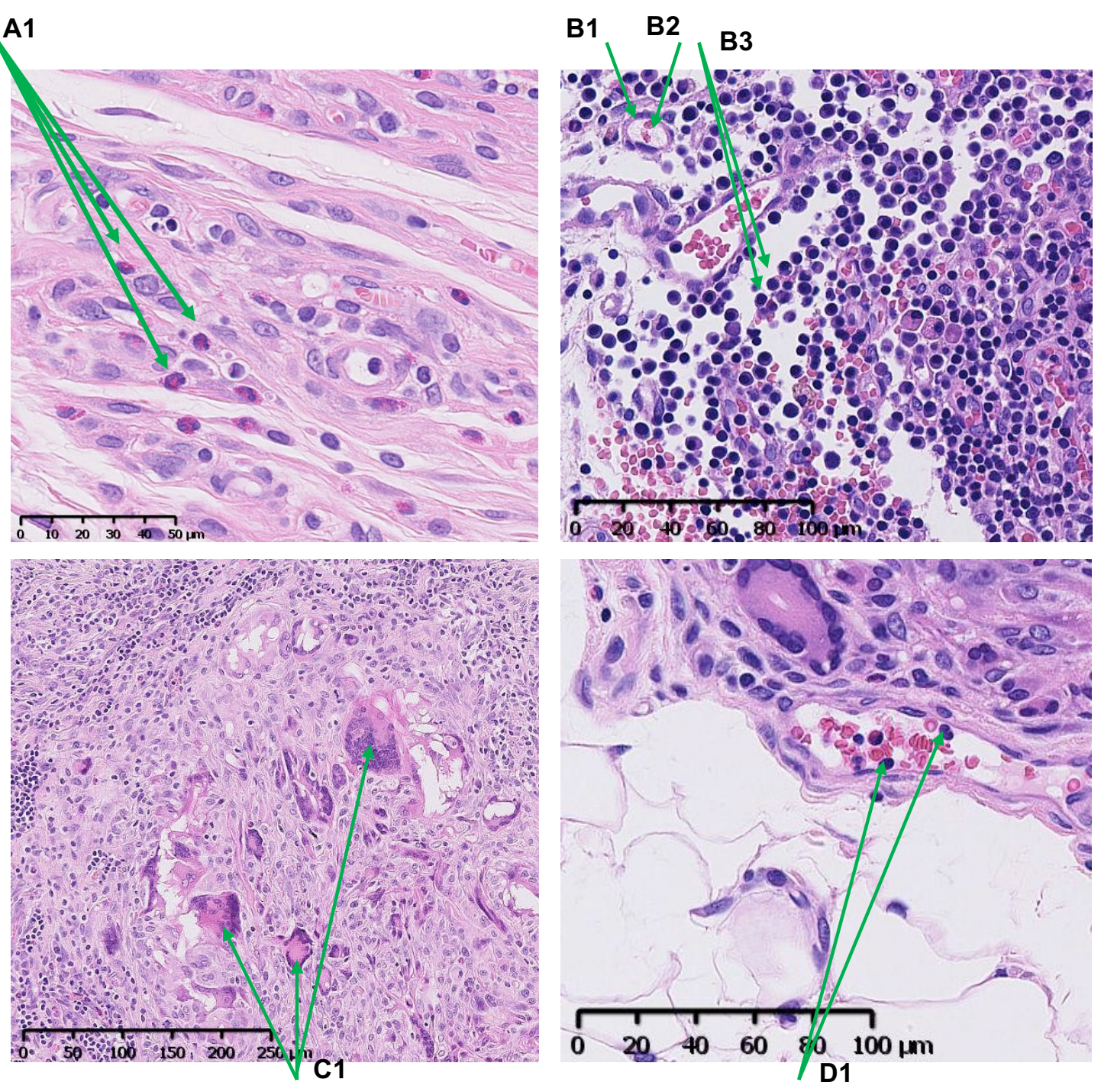

Supplementary 10 Images illustrating the cell types chosen for histological analysis; (a) A1-

Eosinophils (b) B3-Lymphocytes, also shown B1- Blood Vessel and B2-Erythrocyte (c) C1-

Foreign Body Giant Cells (d) D1-Monocytes. All images are stained with H \& E. 
(a)

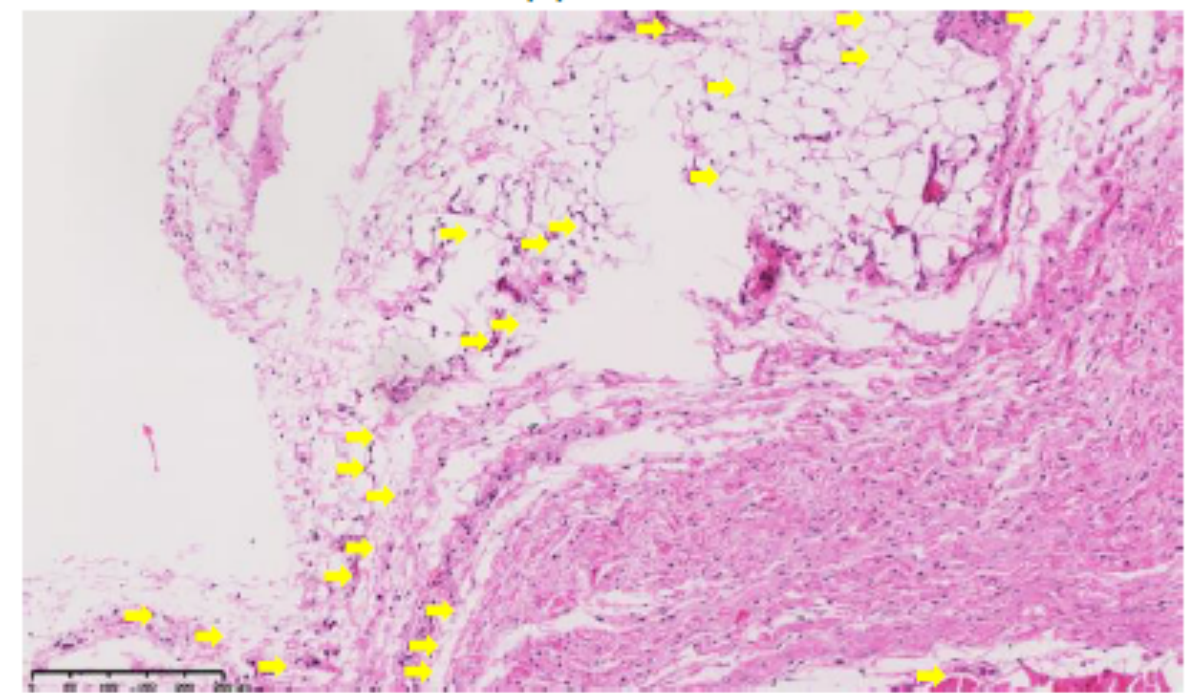

(b)

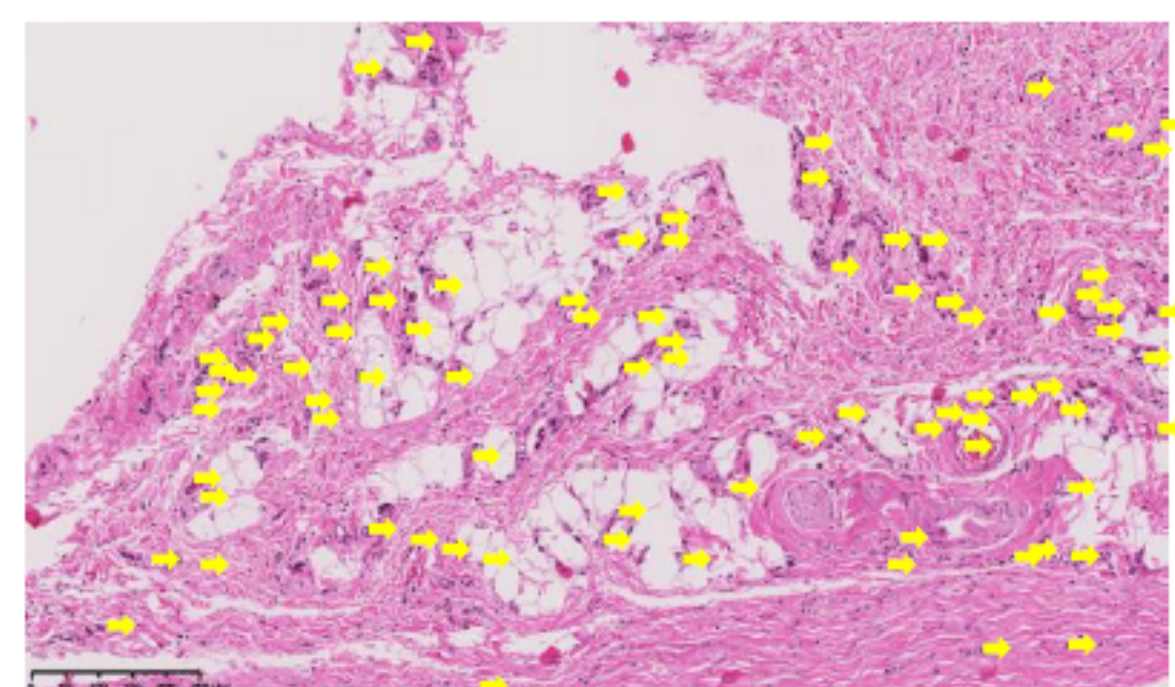

Supplementary 11: Images contrasting the difference in the of density of blood vessels in surrounding granulation tissues for samples with identical fibres and time-points and differing only in the presence of VEGF (a) 5000 rpm at Day 14 without VEGF and (b) $5000 \mathrm{rpm}$ Day 14 with VEGF. Note: The yellow arrows indicate the presence of a blood vessel and may overlay rather than point to the vessel itself. 